\title{
The 3D structure of the Small Magellanic Cloud
}

\author{
Smitha Subramanian ${ }^{1,2}$, Annapurni Subramaniam ${ }^{1}$ \\ Indian Institute of Astrophysics, Koramangala II Block, Bangalore - 34, India \\ Department of Physics, Calicut University, Calicut, Kerala, India
}

\begin{abstract}
The 3D structure of the inner Small Magellanic Cloud (SMC) is investigated using the red clump (RC) stars and the RR Lyrae stars (RRLS), which represent the intermediate-age and the old stellar populations of a galaxy. The V and I bands photometric data from the Optical Gravitational Lensing Experiment (OGLE III) catalog are used for our study. The mean dereddened $\mathrm{I}_{0}$ magnitude of the $\mathrm{RC}$ stars and the RRLS are used to study the relative positions of the different regions in the SMC with respect to the mean SMC distance. This shows that the northeastern part of the SMC is closer to us. The line of sight depth (front to back distance) across the SMC is estimated using the dispersion in the $\mathrm{I}_{0}$ magnitudes of both the RC stars and the RRLS and found to be large $(\sim 14 \mathrm{kpc})$ for both the populations. The similarity in their depth distribution suggest that both these populations occupy a similar volume of the SMC. The surface density distribution and the radial density profile of the RC stars suggest that they are more likely to be distributed in a nearly spheroidal system. The tidal radius estimated for the SMC system is $\sim 7-12 \mathrm{kpc}$. An elongation along the NE-SW direction is seen in the surface density map of the RC stars. The surface density distribution of the RRLS in the SMC is nearly circular. Based on all the above results the observed structure of the SMC, in which both the RC stars and RRLS are distributed, is approximated as a triaxial ellipsoid. The parameters of the ellipsoid are obtained using the inertia tensor analysis. We estimated the axes ratio, inclination of the longest axis with the line of sight $(i)$ and the position angle $(\phi)$ of the longest axis of the elliposid on the sky from the analysis of the RRLS. The analysis of the red clump stars with the assumption that they are extended up to a depth of 3.5 times the sigma (width of dereddened $\mathrm{I}_{0}$ magnitude distribution, corrected for intrinsic spread and observational errors), was also found to give similar axes ratio and orientation angles. The above estimated parameters depend on the data coverage of the SMC. Using the RRLS with equal coverage in all the three axes (data within 3 degrees in $\mathrm{X}, \mathrm{Y}$ and $\mathrm{Z}$ axes), we estimated an axes ratio of $1: 1.33: 1.61$ with $i=2^{\circ} .6$ and $\phi=70^{\circ} .2$. Our tidal radius estimates and the recent observational studies suggest that the full extent of the SMC in the XY plane is of the order of the front to back distance estimated along the line
\end{abstract}


of sight. These results suggest that the structure of the SMC is spheroidal or slightly ellipsoidal. We propose that the SMC experienced a merger with another dwarf galaxy at 4-5 Gyr ago, and the merger process was completed in another 2-3 Gyr. This resulted in a spheroidal distribution comprising of stars older than $2 \mathrm{Gyr}$.

Subject headings: Magellanic Clouds, galaxies: structure, Stars: variable stars, horizontal branch stars

\section{Introduction}

The Small Magellanic Cloud (SMC) located at a distance of around $60 \mathrm{kpc}$ is one of the nearest galaxies. The SMC is characterized by a less pronounced bar, than that seen in the Large Magellanic Cloud (LMC). It also has an eastern extension called the Wing. The Wing and the northeastern part of the bar are closer than the southern parts (Hatzidimitriou et al.|1993). A large line of sight depth was found in the outer and inner regions of the SMC by Gardiner \& Hawkins (1991) and Subramanian \& Subramaniam (2009) respectively.

In an extensive survey of variable stars in the SMC, Graham (1975) discovered 76 RR Lyrae stars (RRLS) in the field centered on the globular cluster, NGC 121. He found that the period distribution of the RRLS in the SMC is unlike that found anywhere in the Galaxy and closely resembles the distribution of variable stars in the Leo II dwarf galaxy. His studies also showed that these stars are distributed rather evenly and concentrate neither towards the bar nor towards the center of the SMC. He mentioned that the lack of strong stellar concentration is another property in common with the stellar populations in the dwarf spheroidal galaxies. Smith et al. (1992) and Soszyñski et al. (2002) also found an even and smooth distribution of RRLS in the northeastern regions and the central 2.4 square degree regions of the SMC respectively. Subramanian \& Subramaniam (2009) used the RRLS data published by Soszyñski et al. (2002) to estimate the depth in the central regions of the SMC. They found that the depth estimated using the red clump (RC) stars and the RRLS are similar in the central regions of the SMC. They suggested that the RRLS and the $\mathrm{RC}$ stars in the central bar region of the SMC are born in the same location and occupy a similar volume in the galaxy. Soszyñski et al. (2010) presented a catalog of the RRLS in the SMC from Optical Gravitational Lensing Experiment (OGLE) III survey. From the spatial distribution of the RRLS they suggested that the halo of the SMC is roughly circular in the sky. However their density map of the RRLS revealed two maxima near the center of the SMC.

Zaritsky et al. (2000) showed that the older stellar populations (age > 1 Gyr) in the SMC are distributed in a regular, smooth ellipsoid. Similar conclusions were drawn by Cioni et al. (2000) from the DENIS near infrared survey. Maragoudaki et al. (2001) further investigated the dynamical 
origin of the bar using isodensity contour maps of stars with different ages. They found similar results for old stellar populations. A sample of 12 populous SMC clusters which possess RC stars are studied by Crowl et al. (2001) to determine the distances to these clusters. The line of sight depth of the SMC is estimated as the standard deviation (sigma) of these distances. They found a 1-sigma depth of $\approx 6-12 \mathrm{kpc}$ for the SMC. Viewing the SMC as a triaxial ellipsoid with RA, DEC and the line of sight depth as the three axes, they found an axes ratio of 1:2:4. From a spectroscopic study of 2046 red giant stars Harris \& Zaritsky (2006) found that the older stellar components of the SMC have a velocity dispersion of $27.5 \mathrm{kms}^{-1}$ and a maximum possible rotation of $17 \mathrm{kms}^{-1}$. Their result is consistent with other kinematical studies based on the radial velocities of the PNe and carbon stars, which represent the old and intermediate-age stellar populations (Dopita et al. 1985, Suntzeff et al. 1986 \& Hatzidimitriou et al. 1997). This implies that the structure of the older stellar component of the SMC is a spheroidal/ellipsoidal, that is supported by its velocity dispersion.

From the analysis of young stars (age < 200 Myr) Zaritsky et al. (2000) suggested that the irregular appearance of the SMC is due to the recent star formation. As in the case of young stars, the large scale HI morphology of the SMC obtained from the high resolution HI observations (Stanimirović et al. 2004) is also quite irregular and does not show symmetry. The most prominent features are the elongation from the northeast to the southwest and the V-shaped concentration in the east. The HI observations also show that the SMC has a significant amount of rotation with a circular velocity of approximately $60 \mathrm{kms}^{-1}$ (Stanimirović et al. 2004) and a large velocity gradient of $91 \mathrm{kms}^{-1}$ in the southwest to $200 \mathrm{kms}^{-1}$ in the north east. (Evans \& Howarth 2008) obtained velocities for 2045 young $(\mathrm{O}, \mathrm{B}, \mathrm{A})$ stars in the SMC, and found a velocity gradient of similar slope as seen in the HI gas. Surprisingly though, they found a position angle $\left(\sim 126^{\circ}\right)$ for the line of maximum velocity gradient that is quite different, and almost orthogonal to that seen in the HI. van der Marel et al. (2009) suggested that this difference in the position angles may be an artifact of the different spatial coverage of the two studies (Evans \& Howarth (2008) did not observe in the North-East region where the HI velocities are the largest), since it would be difficult to find a physical explanation for a significant difference in kinematics between HI gas and young stars. The inclination of the SMC disk in which the young stars are believed to be distributed is estimated as $70^{\circ} \pm 3^{\circ}$ and $68^{\circ} \pm 2^{\circ}$ from the photometric studies of Cepheids by Caldwell \& Coulson (1986) and Groenewegen (2000) respectively. The position angle of the line of nodes is estimated to be $148^{\circ} .0$.

Gardiner \& Noguchi (1996) modelled the SMC as a two component system consisting of a nearly spherical halo and a rotationally supported disc. The tidal radius of the SMC is estimated as $5 \mathrm{kpc}$ according to their model. Their best fit to the distribution halo particles within $5 \mathrm{kpc}$ radius was in good agreement with the observed distributions of the old (>9 Gyr) and intermediate age (2-9 Gyr) stellar populations in the SMC. The distribution of disc particles could reproduce the 
observed irregular distribution of young stars in the SMC. Bekki \& Chiba (2008) suggested that a major merger event in the early stage of the SMC formation caused the coexistence of a spheroidal stellar component and an extended rotating HI disk.

Both the observational and theoretical studies suggest that the old and the intermediate-age stellar populations in the SMC are distributed in a spheroidal/ellipsoidal component. Motivated with this result, here we study the RC stars and the RRLS in the inner SMC, which represent the intermediate-age and the old stellar populations respectively. This study aims to understand the structure of the inner SMC and hence to quantitatively estimate the structural parameters.

The RC stars are core helium burning stars, which are metal rich and slightly more massive counter parts of the horizontal branch stars. They have tightly defined colour and magnitude, and appear as an easily identifiable component in the colour magnitude diagrams (CMDs). These properties of the RC stars make them a good proxy to determine the distance to a galaxy and also to understand the structure of a galaxy. Previously many people have used the RC stars as a proxy for distance estimation (Stanek et al. 1998), to identify the structures in the LMC (Subramaniam 2003) and to estimate the orientation measurements of the LMC (Olsen \& Salyk 2002 and Subramanian \& Subramaniam 2010). Subramanian \& Subramaniam (2009) estimated the depth of both the LMC \& the SMC using the dispersions in magnitude and colour distributions of the RC stars.

The RRLS are metal poor, low mass, core helium burning stars which undergo radial pulsations. Their period of pulsation have a range of 0.2-1 day. The RRLS are excellent tracers of the oldest observable population of stars in a galaxy. The RRab type stars have constant mean magnitude and they are used for distance estimation (Borissova et al. 2009). The dispersion about the mean magnitude is a measure of the depth of the host galaxy. Subramaniam (2006) and Subramanian \& Subramaniam (2009) used the dispersion of the RRab stars to estimate the line of sight depth of the LMC and SMC respectively. Pejcha \& Stanek (2009) studied the structure of the LMC stellar halo using the RRLS in the LMC.

In this paper we estimate the structural parameters of the older component of the SMC from the analysis of the RC stars and the RRLS in the inner SMC. Udalski et al. (2008) presented the $\mathrm{V}$ and I bands photometric data of the 16 sq.degrees of the SMC from the OGLE III survey. The catalog of the SMC RRLS from the OGLE III survey is presented by Soszyñski et al. (2010). Both these data sets are used in this study. The mean dereddened $\mathrm{I}_{0}$ magnitude of the RC stars and the RRLS are used to estimate the relative locations of different regions in the SMC with respect to the mean SMC distance. The dispersion in the mean magnitude is used to obtain the line of sight depth across the SMC.

The structure of the paper is as follows. The next section deals with the contribution of 
heterogeneous population on the dereddened $\mathrm{I}_{0}$ magnitude of the RC stars and RRLS and also to the dispersion in their mean magnitude. In section 3 we explain the data and analysis of both the RC stars and the RRLS. The reddening map of the SMC is presented in section 4. The results of our analysis are given in section 5. The estimation of the structural parameters of the SMC are explained in detail in section 6 . The discussion and the conclusions are presented in section $7 \& 8$ respectively.

\section{Contribution of heterogeneous population}

Stanek et al. (1998) determined the distance to the Magellanic Clouds (MCs) as well as to the bulge of our Galaxy from the dereddened apparent magnitude of the RC stars assuming a constant absolute I band magnitude for them. Later Sarajedini (1999) and Cole (1998) claimed that the luminosity of the RC stars is highly influenced by the age and metallicity and must be accounted for in the determination of the distance. When the controversy regarding the use of RC stars as the absolute indicator continued, they were used to estimate relative distances between regions within MCs. Olsen \& Salyk (2002), Subramaniam (2003) and Subramanian \& Subramaniam (2010) used them as relative distance indicators in their studies.

The RC stars in the SMC are a heterogeneous population and hence, they would have a range in mass, age and metallicity. The density of stars in various location will also vary with the local star formation rate as a function of time. These factors would contribute to the range of magnitude and colour of the net population of the RC stars in any given location of the SMC and hence to the observed peak magnitude and dispersion. Giraradi \& Salaris (2001) simulated the RC stars in SMC using star formation history and age metallicity relation from Pagel \& Tautvaisiene (1998). They created the synthetic CMDs of the SMC and the distributions of the RC stars were fitted using numerical analysis to obtain the mean and dispersion of the magnitude and colour distributions. The model predicted values are used in our study to account for the population effects of the RC stars. The estimated intrinsic widths of the colour and magnitude distributions of the RC stars in the SMC are 0.03 mag and 0.076 mag respectively.

The RRLS in the SMC are also a heterogeneous population with a range in age, metallicity and mass. There are small variations in their luminosities due to evolutionary effects. These factors could contribute to the observed dereddened magnitudes of these stars and also to the dispersion of magnitude within a sample. This is discussed in section 3.2.

Apart from multiple population in a given region, there could be variation in population and metallicity across the SMC. Cioni et al (2006) did not find any different population or metallcity gradient near the central regions of the SMC. Tosi et al (2008) obtained deep CMDs of 6 SMC 
inner regions to study the star formation history. The interesting fact they found about the six CMDs is the apparent homogeneity of old stellar population, populating the subgiant branch and the clump. This suggested that there is no large differences in age and metallicity exist among old stars in the inner SMC.

\section{Data and Analysis}

\subsection{Red Clump Stars}

The OGLE III survey (Udalski et al. 2008) presented the V and I bands photometric data of $16 \mathrm{deg}^{2}$ of the SMC consisting of about 35 million stars. We divided the observed region into 1280 regions with a bin size of $8.88 \times 4.44$ square arcmin. The average photometric error of red clump stars in the I and V bands are around 0.05 mag. Photometric data with errors less than $0.15 \mathrm{mag}$ are considered for the analysis. For each sub-region the (V-I) vs I colour magnitude diagram (CMD) is plotted and the RC stars are identified. A sample CMD is shown is figure 1. For all the regions, red clump stars are found to be located well within the box shown in the CMD, with boundaries 0.65 - $1.35 \mathrm{mag}$ in $(\mathrm{V}-\mathrm{I})$ colour and 17.5 - $19.5 \mathrm{mag}$ in I magnitude. The number of the RC stars identified in each sub-region ranges from 100-3000.

The RC stars occupy a compact region in the CMD. Their number distribution profiles resemble a Gaussian. The peak values of their colour and magnitude distributions are used to obtain the mean dereddened RC magnitude and hence the mean line of sight distance to each sub-region in the SMC. The dispersions in the magnitude and colour distribution of the RC stars are used to obtain the line of sight depth of each sub-region in the SMC.

To obtain the number distribution of the RC stars, they are binned in colour and magnitude with a bin size of 0.01 and 0.025 mag respectively. These distributions are fitted with a Gaussian + Quadratic polynomial. The Gaussian represents the RC stars and the other terms represent the red giants in the region. A nonlinear least-square method is used to fit the profile and to obtain the parameters. The colour and magnitude distributions along with the fitted profiles of a sub-region in the SMC are shown in the lower left panels of figure 2 and figure 3 respectively. The parameters obtained are the coefficients of each term in the function used to fit the profile, error in the estimate of each parameter, and reduced $\chi^{2}$ value. For all the sub-regions we estimated the peaks and the widths in the I mag and (V-I) mag of the distributions, associated errors with the parameters, and reduced $\chi^{2}$ values. The errors associated with the parameters are obtained using the covariance matrix, where the square root of the diagonal elements of the matrix gives the error values.

The number of the RC stars identified within the box in the CMD are less (100-400) for 602 sub-regions. These sub-regions are located towards the edge of the survey and in the three 


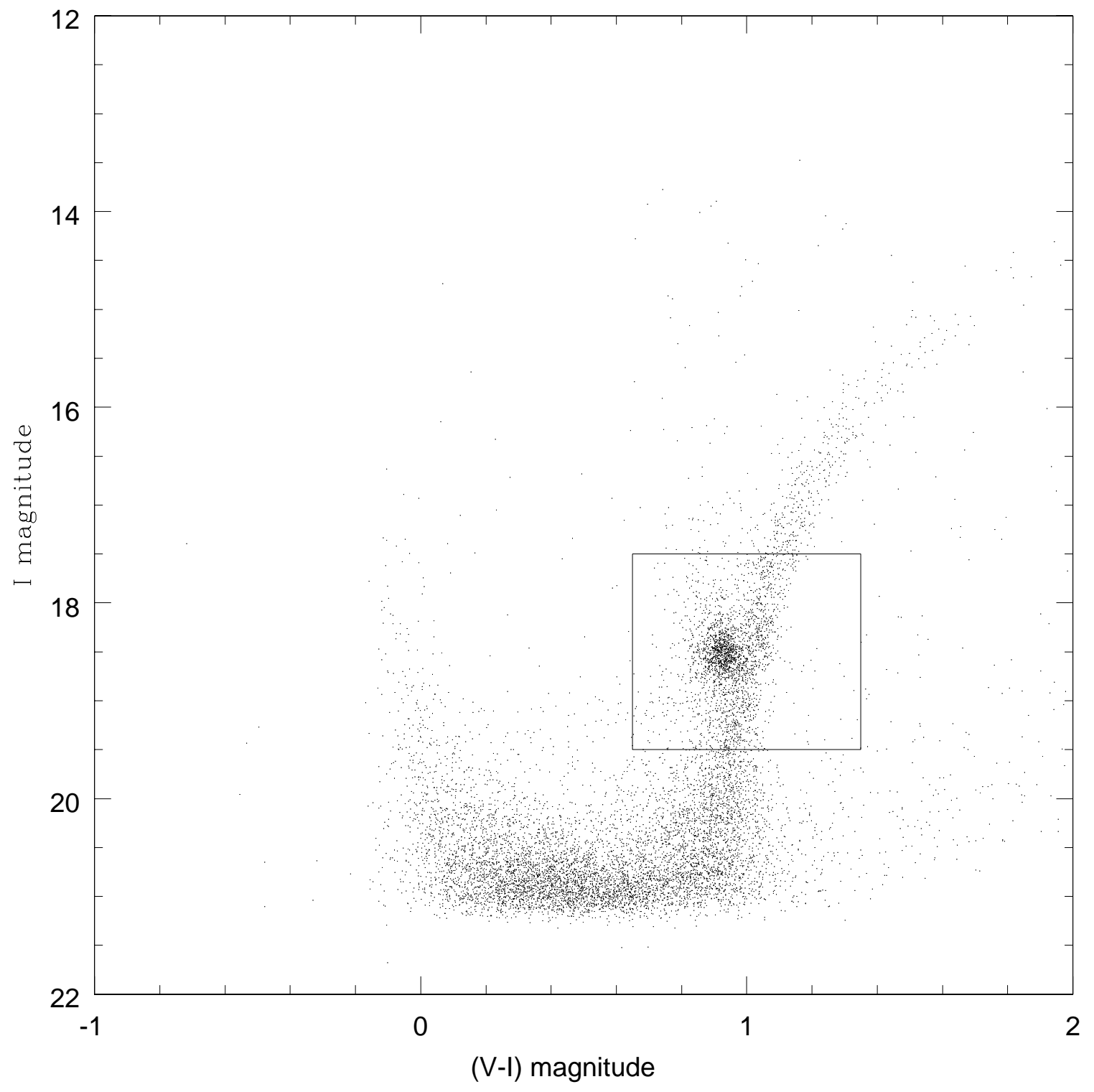

Fig. 1.- The colour magnitude diagram of a sub-region in the SMC. The box used to identify the red clump stars is also shown. 
isolated fields in the north western region of the SMC. For these 602 sub-regions we carefully analyzed the magnitude and colour distributions. Because of the less number of stars the peaks of the distributions were not very clear and unique. It was difficult to fit those distributions with the profiles. As our methodology depends very much on the statistical analysis of the sample we omitted these 602 sub-regions, with RC stars less than 400, for the remaining analysis. Thus the number of regions used for the study reduced to 678. The magnitude and colour distributions of most of these 678 regions were checked manually and found that the fits are satisfactory.

The reduced $\chi^{2}$ values and the fit errors of the peak and width of colour and magnitude distributions are plotted against RA in the lower right, upper right and upper left panels of figure 2 and figure 3 respectively. The sub-regions with reduced $\chi^{2}$ values greater than 2.0 for both magnitude and colour distributions are omitted from the analysis. The regions with peak and width error greater than $0.05 \mathrm{mag}$ in the magnitude distributions are also removed from the analysis. In the case of colour distribution, regions with peak and width error greater than 0.02 mag are not considered for the analysis. Thus we used only 553 sub-regions out of 678 sub-regions, for the final analysis.

The peak value of the colour, (V-I) mag at each location is used to estimate the reddening. The reddening is calculated using the relation $\mathrm{E}(\mathrm{V}-\mathrm{I})=(\mathrm{V}-\mathrm{I})_{o b s}-0.89$ mag. The intrinsic colour of the RC stars in the SMC is chosen as 0.89 mag to produce a median reddening equal to that measured by Schlegel et al. (1998) towards the SMC. The interstellar extinction is estimated by $\mathrm{A}_{I}$ $=1.4 \mathrm{xE}(\mathrm{V}-\mathrm{I})$ (Subramaniam 2005). After correcting the mean I mag for interstellar extinction, $\mathrm{I}_{0}$ mag for each region is estimated.

The variation in the $\mathrm{I}_{0}$ mag between the sub-regions is assumed only due to the difference in the relative distances. The difference in $\mathrm{I}_{0}$ mag is converted into relative distance, $\Delta \mathrm{D}$ using the distance modulus formula,

$$
\left(\mathrm{I}_{0} \text { mean }-\mathrm{I}_{0} \text { of each region }\right)=5 \log _{10}\left(\mathrm{D}_{0} /\left(\mathrm{D}_{0} \pm \Delta \mathrm{D}\right)\right.
$$

where $\mathrm{D}_{0}$ is the mean distance to the $\mathrm{SMC}$ which is taken as $60 \mathrm{kpc}$. The average error in $\mathrm{I}_{0}$ is calculated using the formula, $\delta \mathrm{I}_{0}{ }^{2}=(\text { avg error in peak } \mathrm{I})^{2}+(1.4 \mathrm{x} \text { avg error in peak }(\mathrm{V}-\mathrm{I}))^{2}$, and the error is estimated as 0.013 mag which corresponds to $\sim 360 \mathrm{pc}$.

The cartesian coordinates corresponding to each sub-region can be obtained using the RA, Dec \& $\Delta \mathrm{D}$. The $\mathrm{x}$ axis is antiparallel to the RA axis, $\mathrm{y}$ axis parallel to the declination axis and the $\mathrm{z}$ axis towards the observer. The origin of the system is the centroid of our sample. The centroid estimated as the mean of RA and Dec of the final sample of the RC stars in the 553 sub-regions of the SMC is $\alpha=0^{h} 52^{m} 34^{s} .2 \delta=-73^{\circ} 2^{\prime} 48^{\prime \prime}$. The x,y, and z coordinates 

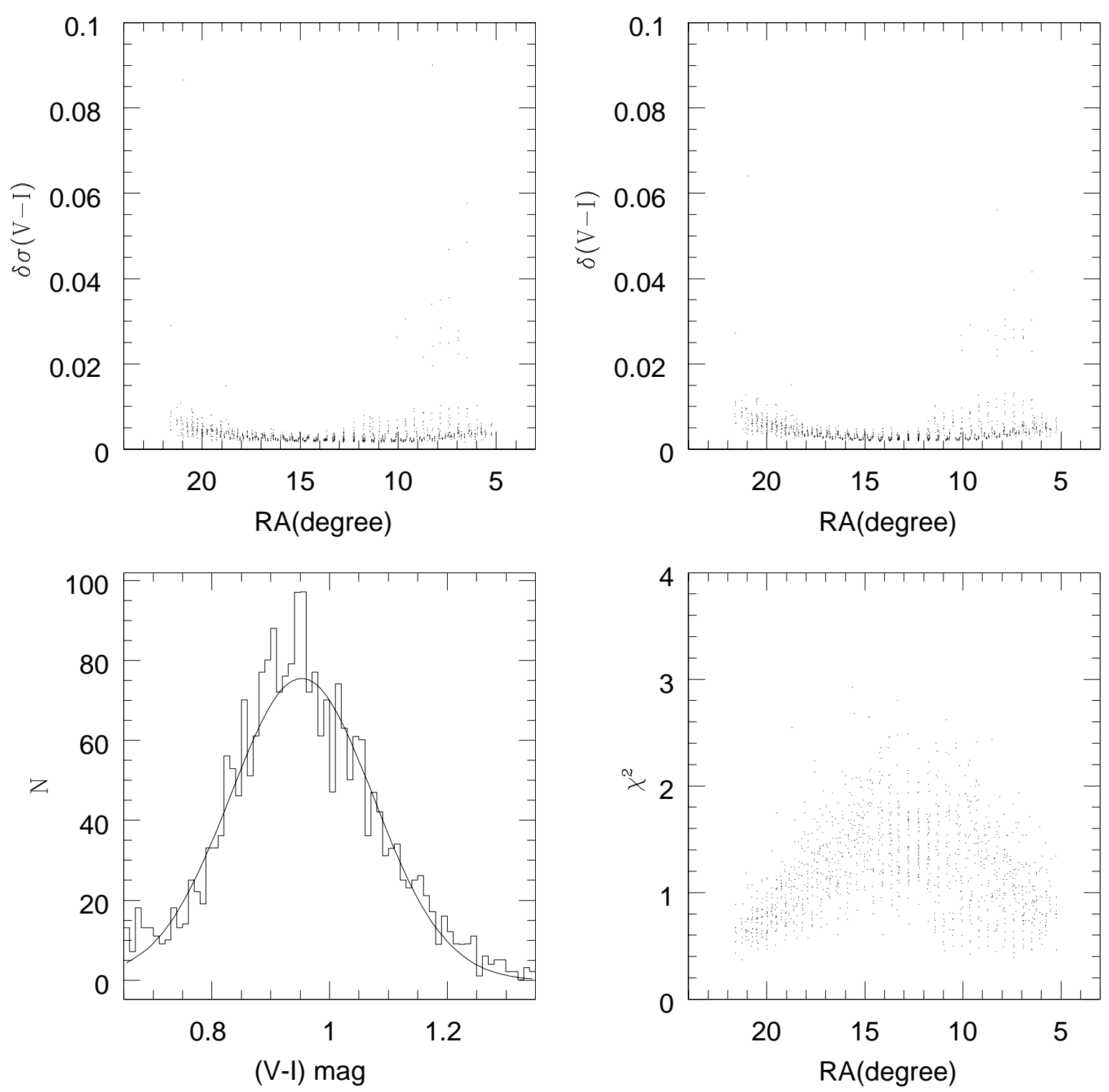

Fig. 2.- A typical colour distribution of a sub-region is shown in the lower left panel. The best fit to the distribution is also shown. The reduced $\chi^{2}$ values and the fit errors in the peak and width of the colour distribution of all sub-regions are plotted against RA in the lower right, upper right and upper left panels respectively. 

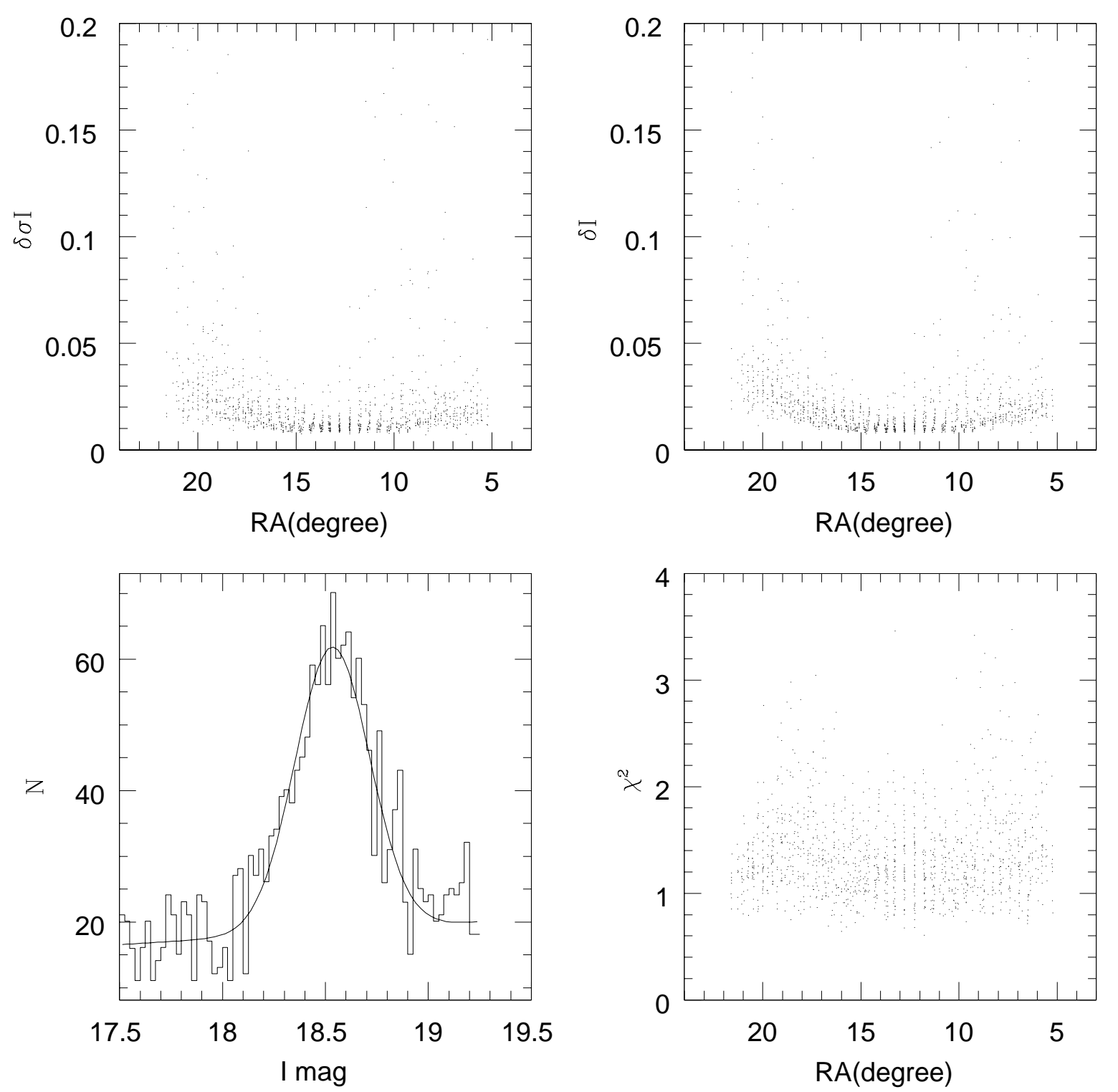

Fig. 3.- A typical magnitude distribution of a sub-region is shown in the lower left panel. The best fit to the distribution is also shown. The reduced $\chi^{2}$ values and the fit errors in the peak and width of the magnitude distribution of all the sub-regions are plotted against RA in the lower right, upper right and upper left panels respectively. 
are obtained using the transformation equations given below van der Marel \& Cioni 2001, see also Appendix of Weinberg \& Nikolaev 2001, similar transformation for the LMC is given in Subramanian \& Subramaniam 2010).

$\mathrm{x}=-\mathrm{D} \sin \left(\alpha-\alpha_{0}\right) \cos \delta$

$\mathrm{y}=\mathrm{D} \sin \delta \cos \delta_{0}-\mathrm{D} \sin \delta_{0} \cos \left(\alpha-\alpha_{0}\right) \cos \delta$,

$\mathrm{z}=\mathrm{D}_{0}-\mathrm{D} \sin \delta \sin \delta_{0}-\mathrm{D} \cos \delta_{0} \cos \left(\alpha-\alpha_{0}\right) \cos \delta$,

where $\mathrm{D}_{0}$ is the distance to the SMC and $\mathrm{D}$, the distance to each sub-region is given by $\mathrm{D}=$ $\mathrm{D}_{0} \pm \Delta \mathrm{D}$. The $(\alpha, \delta)$ and $\left(\alpha_{0}, \delta_{0}\right)$ represent the RA and Dec of each sub-region and the centroid of the sample respectively.

The estimated dispersions of the colour and magnitude distributions are used to obtain the line of sight depth of each region in the SMC. The total width of the Gaussian in the distribution of colour is due to internal reddening, apart from observational error and population effects. The width in the distribution of magnitude is due to population effects, observational error, internal extinction and depth. By deconvolving the effects of observational error, extinction and population effects from the distribution of magnitude, an estimate of the depth can be obtained. The contribution due to population effects on the observed dispersions discussed in section 2 are corrected using the model predicted values given by Giraradi \& Salaris (2001). This analysis for the estimation of the depth is similar to that done by Subramanian \& Subramaniam (2009) for the OGLE II and the Magellanic Cloud Photometric Survey (MCPS) regions of the SMC.

$$
\begin{aligned}
& \sigma_{\text {col }}^{2}=\sigma_{\text {internal-reddening }}^{2}+\sigma_{\text {intrinsic }}^{2}+\sigma_{\text {error }}^{2} \\
& \sigma_{\text {internal-extinction }}=1.4 \times \sigma_{\text {internal-reddening }} \\
& \sigma_{\text {mag }}^{2}=\sigma_{\text {depth }}^{2}+\sigma_{\text {internal-extinction }}^{2}+\sigma_{\text {intrinsic }}^{2}+\sigma_{\text {error }}^{2}
\end{aligned}
$$

Thus the width corresponding to the depth of the RC distribution in each sub-region is estimated. The error in the depth estimate which has contributions from the error in the widths of magnitude and colour is also calculated. The average error in the depth estimate is $\sim 400 \mathrm{pc}$. 


\subsection{RR Lyrae stars}

A catalogue of the SMC RRLS from the OGLE III survey is presented by Soszyñski et al. (2010). To study the old stellar population in the SMC we used 1933 fundamental mode RRLS present in the catalog. We removed 29 stars out of 1933 which are brighter than the SMC RRLS and are possible Galactic objects.

The ab type RRLS could be considered to belong to a similar class and hence assumed to have similar properties. The mean magnitude of these stars in the I band, after correcting for extinction effects, can be used for the estimation of distance and the observed dispersion in their mean magnitude is a measure of the depth in their distribution. The reddening obtained using the RC stars (described in the previous sub-section) is used to estimate the extinction to individual RRLS. Stars within each bin of the reddening map are assigned a single reddening and it is assumed that reddening does not vary much within the bin. Thus the extinction corrected $\mathrm{I}_{0}$ magnitude for all the RRLS are estimated. As in the case of the RC stars, the difference in $\mathrm{I}_{0}$ between each RR Lyrae star is assumed to be only due to the variation in their distances and the relative distance, $\Delta \mathrm{D}$, corresponding to each star is estimated. The error in the relative distance estimation of individual RR Lyrae star is basically the error in the extinction correction of $\mathrm{I}_{0}$ mag. The average error in the extinction estimation, obtained from the RC stars, converts to a distance of $\sim 110 \mathrm{pc}$. The location of each RR Lyrae star in the cartesian coordinate system is then calculated using the transformation equations given in the previous section.

Using the dereddened $\mathrm{I}_{0}$ magnitude of each RR Lyrae star, the dispersion in the surveyed region of the SMC can be found. The dispersion is a measure of the depth of the SMC. The data are binned in 0.5 degree in both $\mathrm{x} \& \mathrm{y}$ axes to estimate dispersion. Thus the observed region is divided into bins with an area of 0.25 square degrees. The number of stars in each bin have a range of 10 - 50. The bins with stars less than 10 are removed from our analysis. For each location we estimated the mean magnitude and the dispersion. The estimated dispersion have contributions from photometric errors, range in the metallicity of stars, intrinsic variation in the luminosity due to evolutionary effects within the sample and the actual depth in the distribution of the stars (Clementini et al. 2003). We need to remove the contribution from the first three terms $\left(\sigma_{\text {intrinsic }}\right)$ so that the value of the last term can be evaluated. Clementini et al. (2003) estimated the value of $\sigma_{\text {intrinsic }}$ as $0.1 \mathrm{mag}$ for their sample of 100 RRLS in the LMC. Subramaniam (2006) used a larger sample of RRLS in the LMC from OGLE II catalog and estimated the value of $\sigma_{\text {intrinsic }}$ as 0.15 mag. Both these values are estimated using the globular cluster data in the observed field. In the case of the SMC, there is only one globular cluster and it is not in our observed field. So we used the intrinsic spread estimated for the RRLS in the LMC for the analysis of the RRLS in the SMC. Using both these values we estimated the corrected sigma values which correspond to only depth. The relation used is 


$$
\sigma_{I_{0} m a g}^{2}=\sigma_{\text {depth }}^{2}+\sigma_{\text {intrinsic }}^{2}
$$

\section{Reddening Map of the SMC}

One of the by-product of this study is the estimation of reddening towards the SMC. The observed shift of the peak (V-I) colour of the RC stars in the LMC, from the expected value was used by Subramaniam (2005) to estimate the line of sight reddening map to the OGLE II regions of the LMC. Such a reddening map towards the SMC using the RC stars is obtained here. The intrinsic value of the $(\mathrm{V}-\mathrm{I})$ colour of the RC stars in the SMC is chosen as 0.89 mag to produce a median reddening equal to that measured by Schlegel et al. (1998) towards the SMC. Using this value the $\mathrm{E}(\mathrm{V}-\mathrm{I})$ values are estimated as detailed in section 2.1. The colour coded figure of the reddening in the SMC is presented in figure 4. The colour code is given in the plot. The average value of $\mathrm{E}(\mathrm{V}-\mathrm{I})$ obtained towards the $\mathrm{SMC}$ is $0.053 \pm 0.017 \mathrm{mag}$. From the plot we can see that most of the regions in the SMC have E(V-I) less than 0.08 mag shown as cyan and blue points. The regions in the south western and north eastern sides of the center and the eastern wing regions have larger reddening compared to the other regions of the SMC. This reddening map estimated using the RC stars are used for dereddening the RC stars as well as the RRLS in this study. This reddening data will be made available electronically as an online table.

The previous estimates of the reddening towards the SMC are compared with our estimates. Caldwell \& Coulson (1985) found a mean E(B-V) of 0.054 mag from the analysis of 48 Cepheids. This value can be converted into an $\mathrm{E}(\mathrm{V}-\mathrm{I})$ of 0.0756 mag using the relation $\mathrm{E}(\mathrm{V}-\mathrm{I})=1.4 *$ $\mathrm{E}(\mathrm{B}-\mathrm{V})$ (Subramaniam 2005). Udalski (1998) (using red clump stars) estimated a mean E(V-I) value of $0.1 \mathrm{mag}$. Massey et al. (1995) and Grieve \& Madore (1986) estimated a E(B-V) of around 0.09 mag which converts to an E(V-I) value of 0.126 mag. Haschke et al (2011) provided the reddening maps towards the SMC from the study of the RC stars and RRLS. The intrinsic colour of the RC stars used by them is also $0.89 \mathrm{mag}$. The reddening map of the SMC estimated from the RC stars given in Figure 4 of Haschke et al (2011) is very similar to our reddening map given in Figure 4. The mean value of E(V-I) estimated by them towards the SMC using the RC stars is 0.04 mag and that estimated from RRLS is 0.07 mag. The reddening maps of the RC stars and RRLS given in Figure 4 and Figure 11 of Haschke et al (2011) are very similar. This fact supports and validates our usage of the reddening map obtained from the RC stars for dereddening the RRLS. The previous estimates other than that of Haschke et al (2011) are higher than our estimates. This could be due to the choice of our intrinsic (V-I) colour of the RC stars in the SMC. But such a change only will shift the reddening value in all regions in a similar way. As we are only interested in the relative positions of the regions in the SMC such a change in the intrinsic colour of the RC 
stars is not going to affect our final results.

In order to compare the adopted intrinsic colour of the RC stars in the SMC with theoretical values, we measured the peak colour of the RC stars from the synthetic CMD of the SMC given in Giraradi \& Salaris (2001). It turned out to be $\sim 0.89$ mag. The table given in Giraradi \& Salaris (2001) provide an $\mathrm{RC}(\mathrm{V}-\mathrm{I})_{0}$ colour for the LMC metallicity of $\mathrm{z}=0.004$ between 0.90 and 0.94 mag (age between 2-9 Gyr). For a lower metallicity of $z=0.001$, their models lead to a colour between $0.8 \mathrm{mag}$ and $0.84 \mathrm{mag}$ (age between 2-9 Gyr). The mean metallicity found by Cole (1998) and Glatt et al (2008) for the SMC is between 0.002 and 0.003 . This value supports our adopted value of $(\mathrm{V}-\mathrm{I})_{0}$ colour of the RC stars in the SMC, which is bluer than that of the LMC and redder than the colour obtained for a lower metallicity system.

\section{Results}

\subsection{Relative distances}

The mean dereddened $\mathrm{I}_{0}$ magnitude of the RC stars for the 553 sub-regions of the SMC are estimated. The mean magnitudes of different sub-regions are shown as different colour points in the two dimensional plot of $\mathrm{X}$ vs $\mathrm{Y}$ in the figure 5. The colour code is given in the figure. The average dereddened magnitude, $\mathrm{I}_{0}$ of 553 sub-regions is $18.43 \pm 0.03 \mathrm{mag}$. The regions in the south $(\mathrm{y}<0)$ are fainter compared to the regions in the north. The brighter regions $\left(\mathrm{I}_{0}<18.39\right)$ shown as blue points are located more in the northeastern side. This result indicates that the northeastern regions of the SMC are closer to us.

The relative distance to each RR Lyrae star with respect to the mean distance to the SMC is estimated from the dereddened $\mathrm{I}_{0}$ magnitude, assuming that the average distance to our sample of RRLS in the SMC is $60 \mathrm{kpc}$. The spatial distribution of RRLS in the XZ and YZ planes with over plotted density contours are shown in figure 6 and figure 7 respectively. The convention of the +ve and -ve $\mathrm{Z}$ axes are such that the +ve $\mathrm{Z}$ axis is towards us and -ve $\mathrm{Z}$ axis away from us. The distribution of RRLS in the SMC looks more or less symmetric and extends from $-20 \mathrm{kpc}$ to $+20 \mathrm{kpc}$ with respect to the mean. Most of the stars are located between $\pm 10 \mathrm{kpc}$. An extension to large distances can be seen at $\mathrm{x} \sim 0$. The outer density contours shown in figure 6 indicate that the eastern side of the SMC is on an average closer to us than the western side. We calculated the average $\mathrm{I}_{0}$ magnitude of RRLS in the eastern end $(\mathrm{x}<-1.5,245$ stars $)$ and in the western end $(\mathrm{x}>1.5$, 221 stars). It was found that the eastern side is 0.034 mag brighter than the western side, which makes the eastern side on an average 950 pc closer to us than the western side. Similarly, the outer density contours shown in figure 7 suggest that the northern side is closer to us than the southern side. We calculated the average $\mathrm{I}_{0}$ in the northern end ( $\mathrm{y}>1.5,133$ stars) and in the southern end 


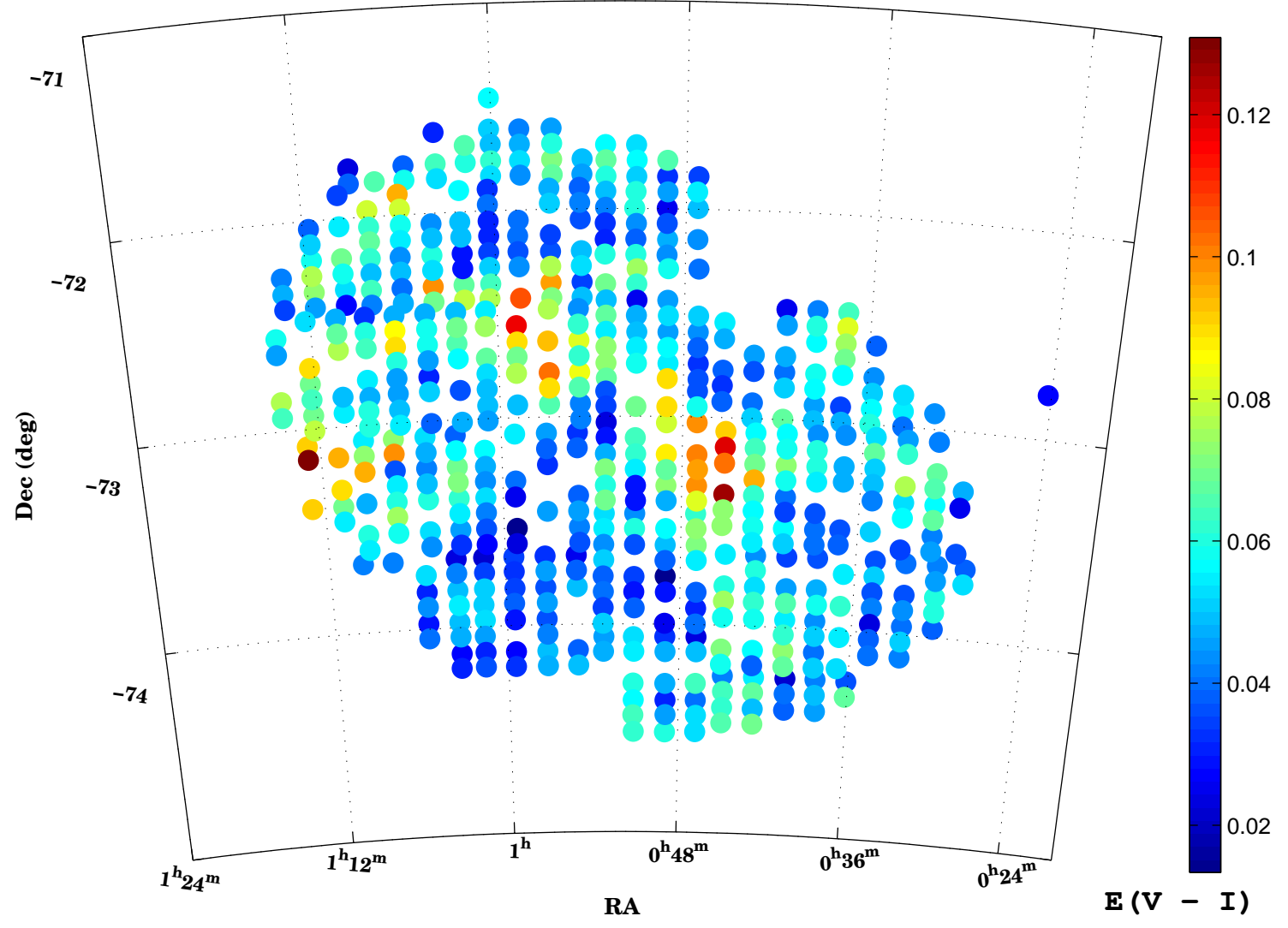

Fig. 4. - A two dimensional plot of the reddening in the sub-regions of the SMC. 


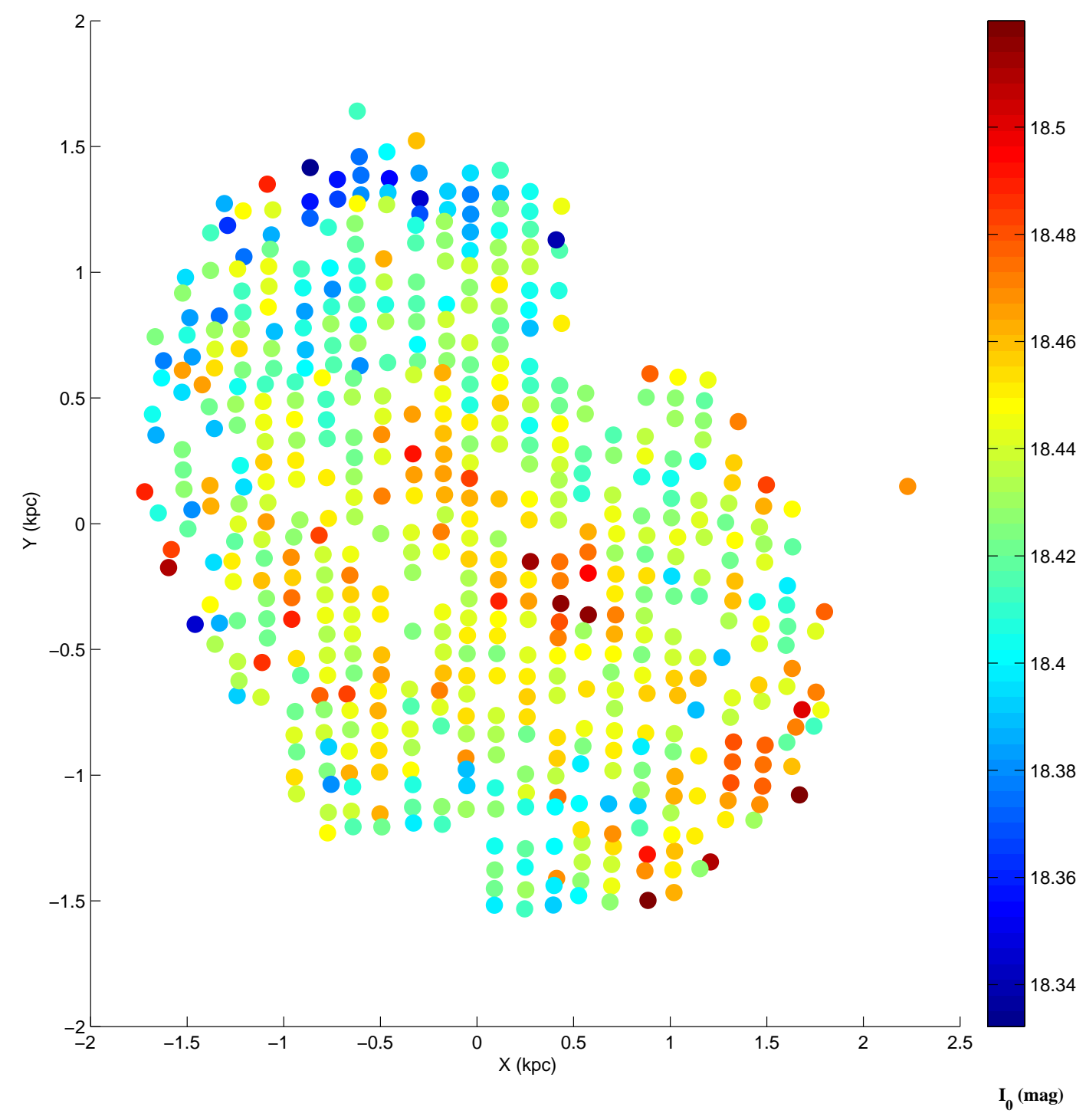

Fig. 5.- A two dimensional plot of the mean magnitudes of the RC stars in the sub-regions of the SMC. 
( $y<-1.5,104$ stars). It was found that the northern side is 0.019 mag brighter than the southern side, which makes the northern side on an average $520 \mathrm{pc}$ closer to us than the southern side. Thus the outer density contours of figures 6 and 7 and the quantitative estimates mildly suggest that the north eastern part of the SMC is closer to us. This result is very similar to the result that obtained from the RC stars. As this effect is significant in outer regions and our study is limited to inner regions, we need more data in the outer region to securely say that the northeastern part of the SMC is closer to us. From the photometric study of stellar populations upto $11.1 \mathrm{kpc}$ in the SMC, Nidever et al (2011) found that the eastern part of the SMC is closer to us than the western side.

In the case of the RC stars as well as RRLS, the variation in the $\mathrm{I}_{0}$ magnitude is assumed as only due to the difference in the distances. As discussed earlier in section 2, there can be contributions in their mean magnitude from the population effects. Thus the line of sight distance estimates suggest that either the RC star and RRLS in the north eastern part of the SMC are different from those found in the other regions of the SMC and/or the northeastern regions of the SMC are closer to us. The previous studies mentioned in section 2 suggest that there is no large variation in the age and metallcity among old stars in the inner SMC. Thus the contribution to the $\mathrm{I}_{0}$ magnitude from the population effects across the SMC is expected to be minimum. The exact contribution can be understood only from the detailed spectroscopic studies of the old stellar populations.

\subsection{Line of sight depth of the SMC}

The dispersions in the magnitude and colour distributions of the RC stars are used to obtain the depth corresponding to the width (1-sigma) of the 553 sub-regions in the SMC. The width corresponding to depth is converted into depth in kpc using the distance modulus formula,

$$
\sigma_{\text {depth }}=5 \log _{10}\left[\left(\mathrm{D}_{0}+\mathrm{d} / 2\right) /\left(\mathrm{D}_{0}-\mathrm{d} / 2\right)\right.
$$

where $\mathrm{d}$ is the line of sight depth in kpc.

A colour coded, two dimensional plot of depth is shown in figure 8 . The colour code is explained in the plot. From the plot we can see that the depth distribution in the SMC is more or less uniform. The prominent feature in the plot is the enhanced depth (depth $>8 \mathrm{kpc}$ ) seen near the central regions. An increased depth of around 6-8 kpc is seen near the north eastern regions also. The average depth obtained for the SMC observed region is $4.57 \pm 1.03 \mathrm{kpc}$. These results

are matching well with the previous depth estimates by Subramanian \& Subramaniam (2009).

The dispersion in the mean magnitude of RRab stars is also used to estimate the depth (1- 


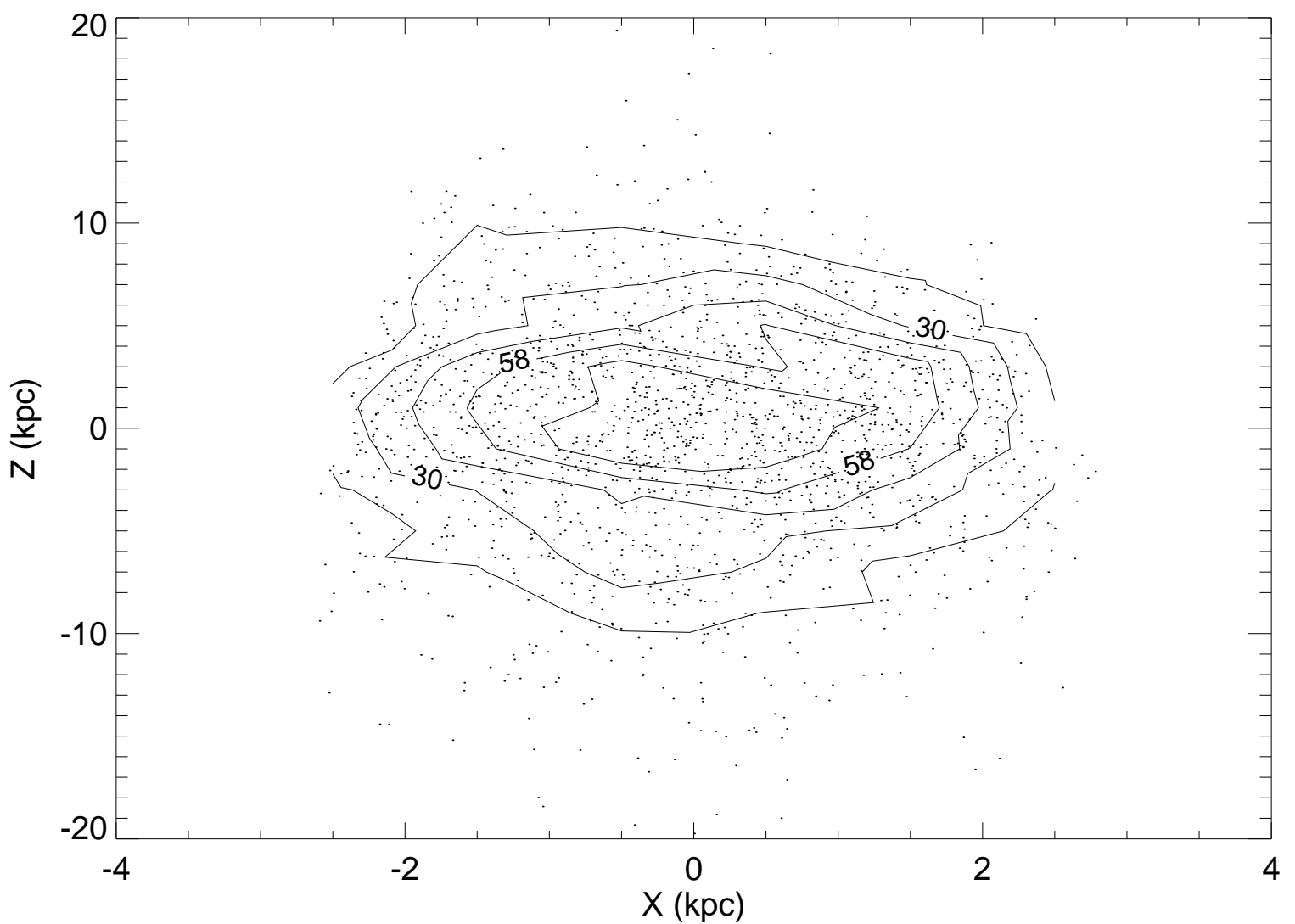

Fig. 6.- Relative distances to each RR Lyrae star in the SMC with respect to the mean distance are plotted against $\mathrm{X}$ axis and are shown as black dots. The density contours are over plotted. The east is in the direction of decreasing $X$ axis. 


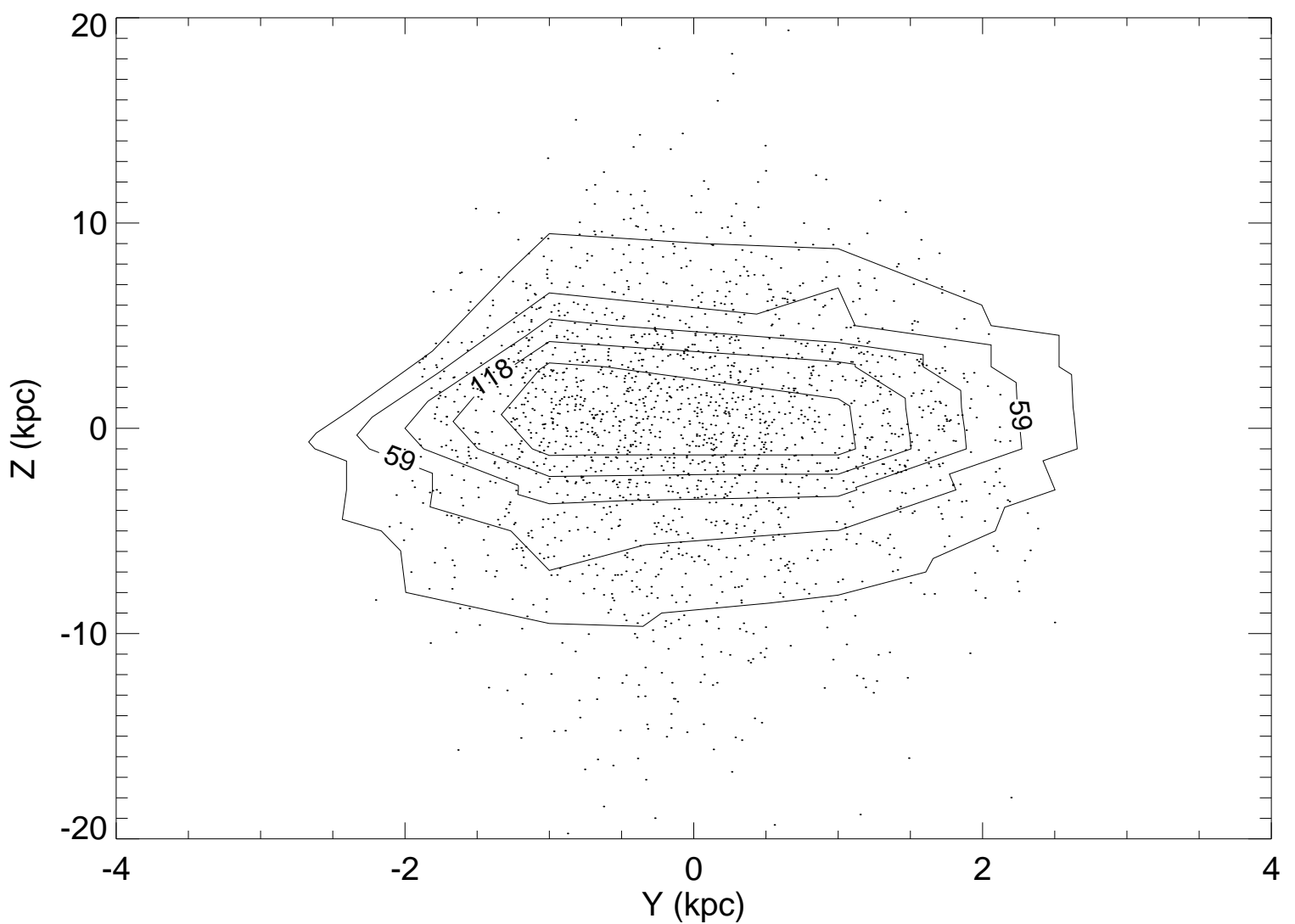

Fig. 7.- Relative distances to each RR Lyrae star in the SMC with respect to the mean distance are plotted against $\mathrm{Y}$ axis and are shown as black dots. The density contours are over plotted. The north is in the direction of increasing $\mathrm{Y}$ axis. 


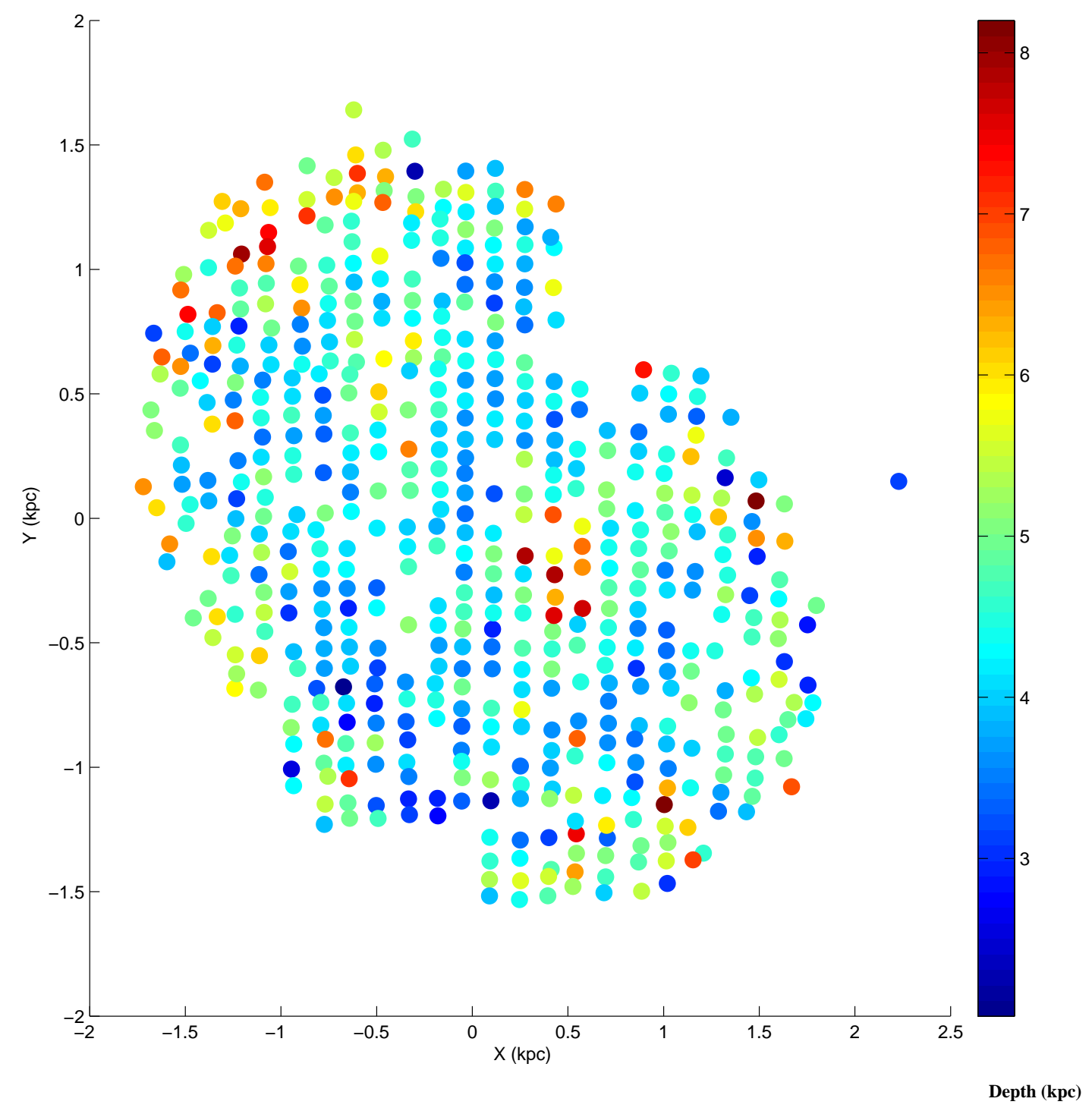

Fig. 8.- A two dimensional plot of the depth in the sub-regions of the SMC obtained from the analysis of the RC stars. 
sigma) of the SMC. The dispersion in the mean magnitude for 70 sub-regions of the SMC is estimated and converted into depth in kpc. The average value of depth estimated when the intrinsic spread was taken as $0.1 \mathrm{mag}$ is $4.07 \pm 1.68 \mathrm{kpc}$ and the average depth is $3.43 \pm 1.82 \mathrm{kpc}$ when 0.15 mag was taken as the intrinsic spread. These estimates match with the depth estimate obtained from the RC stars within the error bars.

The depth estimated using the RC stars and RRLS are plotted together in figure 9 against the $\mathrm{X}$ and $\mathrm{Y}$ axes. In both the lower panels, the depth values are plotted against the $\mathrm{X}$-axis and in both the upper panels, they are plotted against the Y-axis. Basically the depth values correspond to the extent (front to back distance) over which these stars are distributed in the SMC. The measured 1-sigma depth (front to back distance) is halved and plotted along the +ve and -ve depth-axis, assuming that the depth is symmetric with respect to the SMC. The red crosses correspond to the depth estimated using the RC stars and the black points correspond to the depth estimated using the RRLS. The error bars for each point are not plotted to avoid crowding. In the left panels, the black dots correspond to the depth estimated using the RRLS when the $\sigma_{\text {intrinsic }}$ is taken as 0.1 mag. Similarly, in the right panels the black dots correspond to the depth estimated using the RRLS when the $\sigma_{\text {intrinsic }}$ is taken as $0.15 \mathrm{mag}$. The larger depth near the center (x 0 and $\left.\mathrm{y} \sim 0\right)$ is seen for both the populations. The 18 regions in the right panels and the 3 regions in the left panels which are shown as open circles at zero depth are those for which the depth of the RRLS became less than zero when the correction for $\sigma_{\text {intrinsic }}$ were applied. For a large number of regions the depth value becomes less than zero when $\sigma_{\text {intrinsic }}$ is taken as 0.15 mag. Thus, it appears to be more appropriate to take $\sigma_{\text {intrinsic }}$ as $0.1 \mathrm{mag}$ for the RRLS in the SMC. The depth profiles of both the populations are similar, suggesting that both the RC stars and RRLS occupy a similar volume in the SMC.

It is important to see how the dispersion in the distribution of the RRLS is related to the real RRLS distribution, obtained from the individual RRLS distances. This comparison will help us to estimate the actual depth from the dispersion of the RRLS in the SMC. We halved the 1-sigma depth (front to back distance estimated after correcting for $\sigma_{\text {intrinsic }}$ to be taken as $0.1 \mathrm{mag}$ ) obtained for each sub-region with respect to the mean distance and plotted in the -ve and +ve $\mathrm{Z}$ axis as open circles against the $\mathrm{X}$-axis in the left lower panel of figure 10. Similarly, 2-sigma depth,3-sigma depth and 3.5-sigma depth are plotted in the lower right panel, upper-right panel and upper left panel of figure 10 respectively. The individual RRLS distances with respect to the mean SMC distance are shown as black dots in all the panels of figure 10. From the figure we can clearly see that the distribution of RRLS is atleast extended upto 3.5 sigma dispersion, if we take an intrinsic spread of $0.1 \mathrm{mag}$. This 3.5 sigma width $\left(\sigma_{\text {dep }}=0.146 \mathrm{mag}\right)$ translates to a front to back distance of around $14.12 \mathrm{kpc}$. In figure 11, the depth estimated from RRLS after correcting for $\sigma_{\text {intrinsic }}=$ 0.15 mag are plotted over the individual RRLS distances. The left upper panel shows the 4 sigma depth plotted over the individual RRLS distances. From the figure we can clearly see that the 


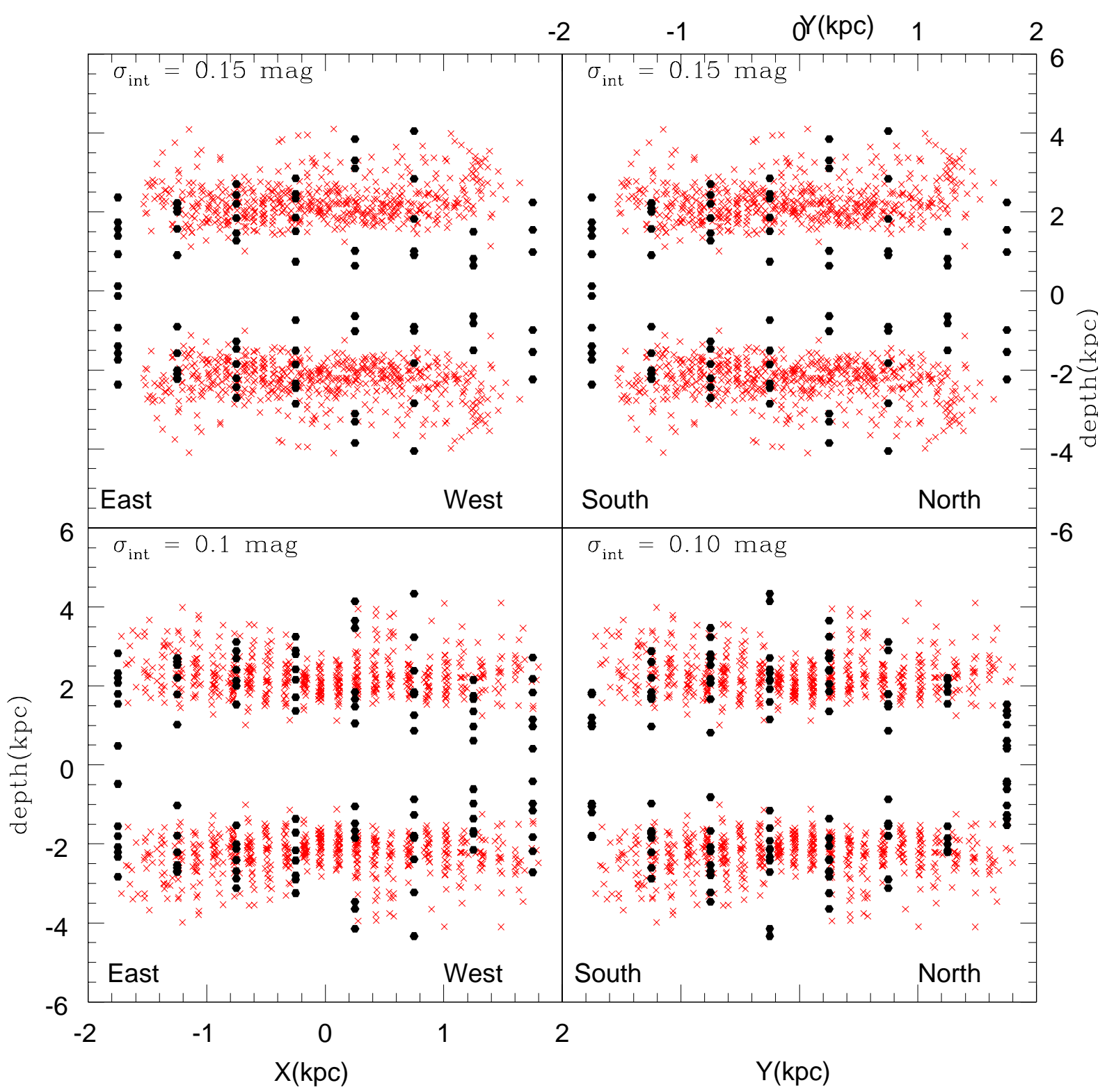

Fig. 9.- The line of sight depth (1-sigma) of both the RC stars (red crosses) and the RRLS (black dots) are plotted against the $\mathrm{X}$ and $\mathrm{Y}$ axes. In the left lower and left upper panels, the RRLS depth estimates are done using $\sigma_{\text {intrinsic }}=0.1 \mathrm{mag}$. In the right lower and left upper panels, the RRLS depth estimates are done using $\sigma_{\text {intrinsic }}=0.15$ mag. 
distribution of RRLS is atleast extended to 4 sigma dispersion, if we take an intrinsic spread of 0.15 mag. This 4 sigma width $\left(\sigma_{\text {dep }}=0.124 \mathrm{mag}\right.$ ) translates into a front to back distance of around $13.7 \mathrm{kpc}$. Thus the RRLS in the SMC are distributed over a distance of $\sim 14 \mathrm{kpc}$ along the line of sight. In the case of the RC stars, we have estimated only the mean distances to the sub-regions and the depth of the sub-regions. So we cannot compare the real RC distribution with the width of the distribution to define the depth. Since the depth profiles of the RC stars and the RRLS are similar, we expect these two populations to occupy a similar volume in the SMC and the RC stars also to be distributed in a depth of $14 \mathrm{kpc}$. The large depth suggests a spheroidal/ellipsoidal distribution for the above populations.

\subsection{Density distributions of $R C$ and RR Lyrae stars}

From the above section we found that both the RC stars and RRLS have similar line of sight depth. The density distributions of both these populations will give a clue about the system in the XY plane. The surface density distribution, and the radial density profile are studied to understand the structure of the SMC. The surface density is calculated by dividing the observed region into different sub-regions and obtaining the number of objects per unit area in each sub-region. The radial density profiles are obtained by finding the projected radial number density of objects in concentric rings around the centroid of the SMC. These profiles can be compared with the theoretical models. The two theoretical models which can be used for the comparison of spatial distribution of different stellar populations in the SMC are the exponential disk profile and the King's profile. The exponential disk profile is give by

$\mathrm{f}(\mathrm{r})=\mathrm{f}_{0 d} \mathrm{e}^{-r / h}$

where $\mathrm{f}_{0 d}$ and the $\mathrm{h}$ represent the central density of the objects and the scale length, respectively and $r$ is the distance from the centre of the distribution. The King's profile (King 1962), which is often used to describe the distribution of globular clusters, but also applies to dwarf spheroidal galaxies, is given by

$\mathrm{f}(\mathrm{r})=\mathrm{f}_{0 k}\left\{\left[1+(\mathrm{r} / \mathrm{a})^{2}\right]^{-1 / 2}-\left[1+\left(\mathrm{r}_{t} / \mathrm{a}\right)^{2}\right]^{-1 / 2}\right\}^{2}$

where $\mathrm{r}_{t}$ and a are the tidal and core radii respectively, $\mathrm{f}_{0 K}$ is the central density of objects and $r$ is the distance from the centre. Both these profiles are used to fit the observed distribution of the $\mathrm{RC}$ stars in the SMC.

The number of the RC stars, identified from the CMD, in each sub-region of the OGLE III 


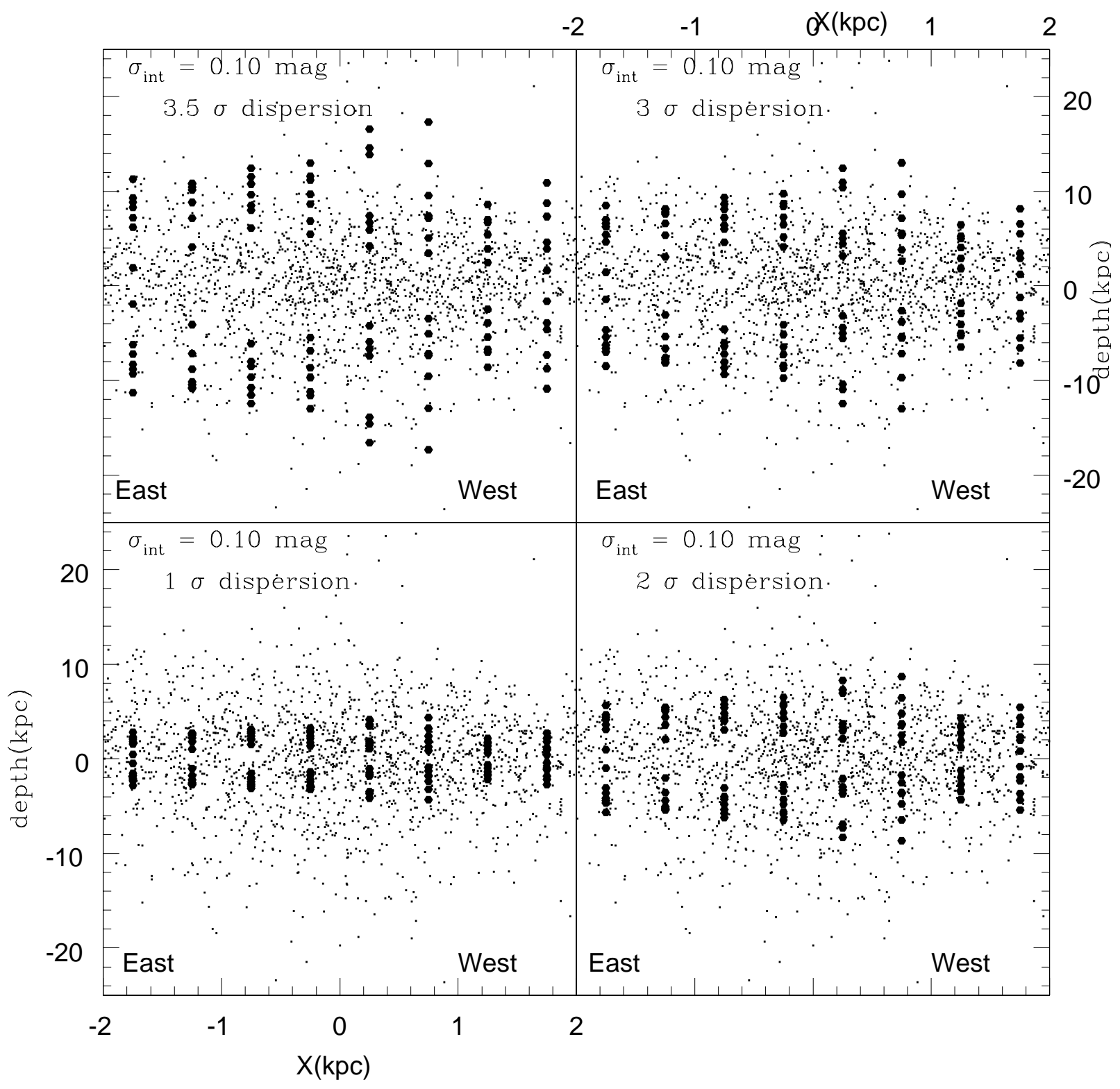

Fig. 10.- The distance to each RRLS with respect to the mean distance to the SMC is plotted as black dots in all the panels against the $\mathrm{X}$-axis. The open circles in all the panels from lower right to upper left in the counter clockwise direction the 1-sigma, 2-sigma, 3-sigma \& 3.5-sigma depth halved with respect to the mean distance to each sub-region and plotted in the -ve and +ve Z-axis against the $\mathrm{X}$ axis. The depth is calculated using an intrinsic spread of 0.1 mag. 


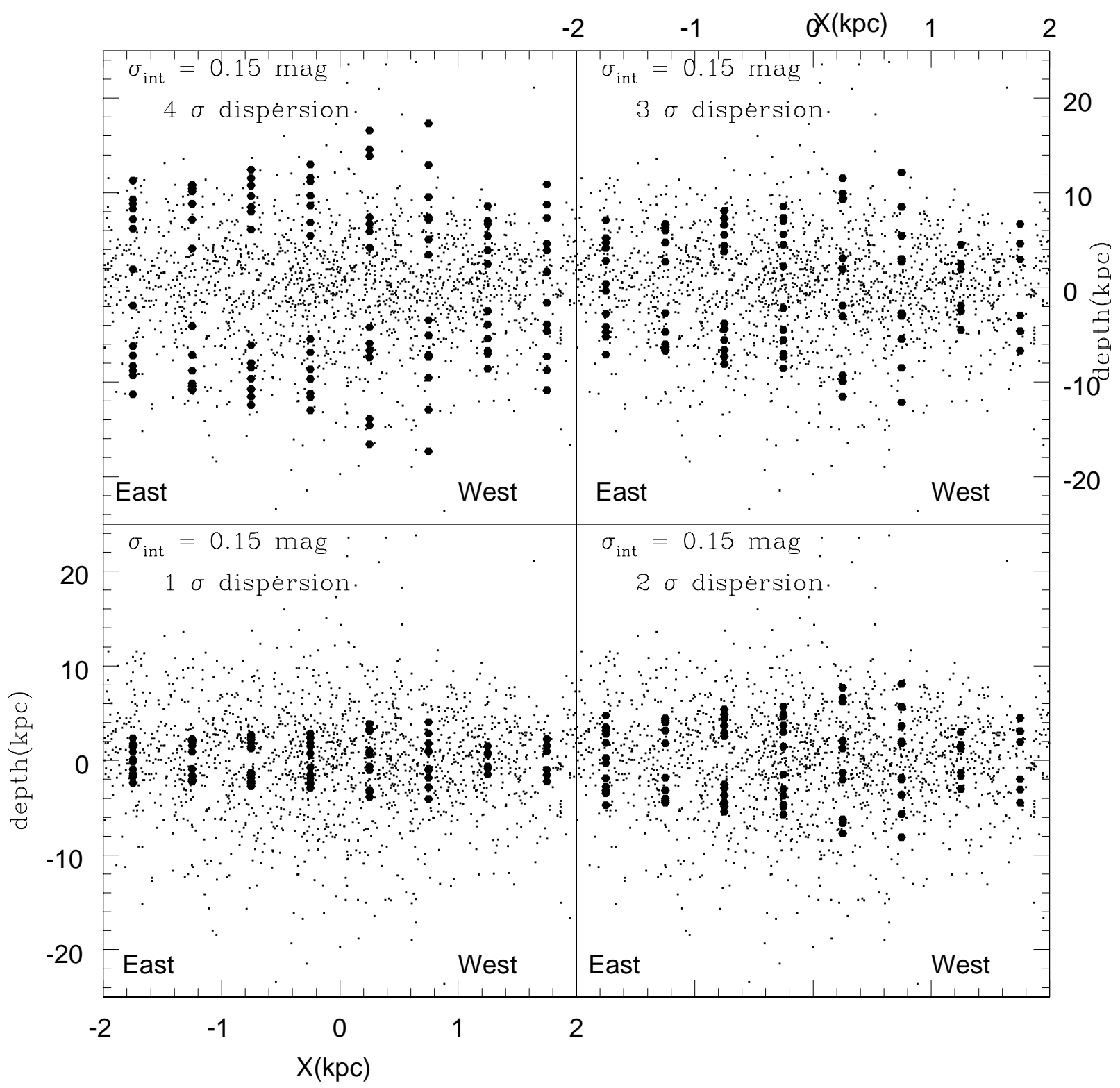

Fig. 11. - The distance to each RRLS with respect to the mean distance to the SMC is plotted as black dots in all the panels against the $\mathrm{X}$-axis. The open circles in all the panels from lower right to upper left in the counter clockwise direction the 1-sigma, 2-sigma, 3-sigma \& 4-sigma depth halved with respect to the mean distance to each sub-region and plotted in the -ve and +ve Z-axis against the $\mathrm{X}$ axis. The depth is calculated using an intrinsic spread of $0.15 \mathrm{mag}$. 


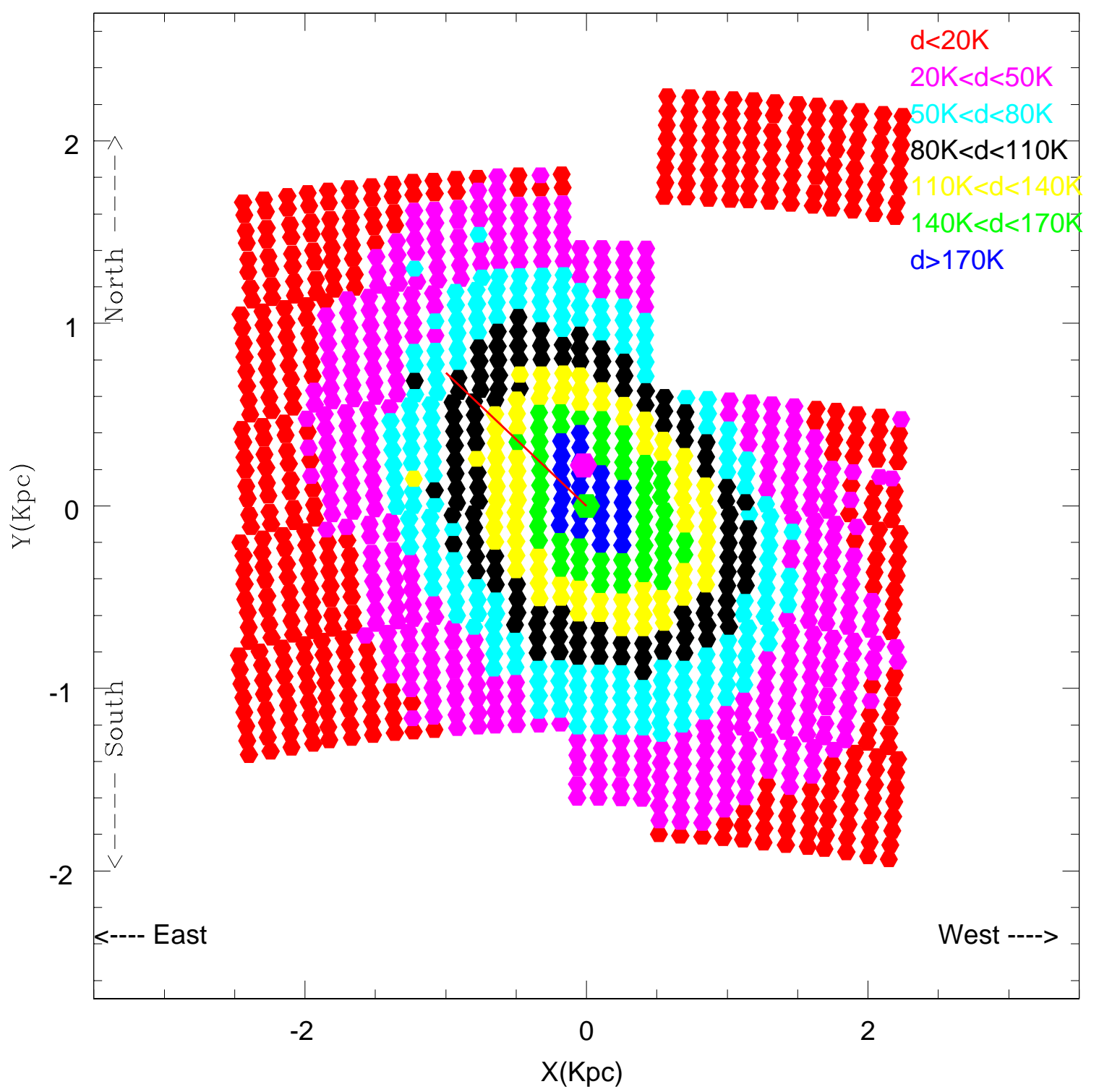

Fig. 12.- A two dimensional plot of the number density,d of the RC stars ( $d$ in the units of $1000(\mathrm{~K}) / \mathrm{square} \mathrm{kpc})$ in the SMC. The green and magenta hexagons represent the centroid of the sample and the optical center respectively. The red line shows the direction of elongation. 
region are estimated. We used all the 1280 sub-regions (each having an area of 32.6 square $\mathrm{kpc}$ ) in the observed OGLE III region. We estimated the number density, number of the RC stars per unit area, for each sub-region. The number density distribution of the RC stars is shown in figure 12 . The number density of RC stars in each sub-region ranges from $7500 / \mathrm{kpc}^{2}-200,000 / \mathrm{kpc}^{2}$. In this figure, discrete points represent each sub-region and the colour denotes the number density. The plot clearly shows the smooth RC distribution with an elongation in the north-east to south- west (NE-SW) direction. The colour code is given in the figure, where d denotes the red clump number density. The position angle of the elongation of the $\mathrm{RC}$ distribution is around $54^{\circ}$ for the eastern side. The plot also shows the shift in the RC density center, $\alpha=0^{h} 52^{m} 34^{s} .2, \delta=-73^{\circ} 2^{\prime} 48^{\prime \prime}$ (shown as green hexagon in figure 12) from the optical center, $\alpha=0^{h} 52^{m} 45^{s}, \delta=-72^{\circ} 49^{\prime} 43^{\prime \prime}$ (shown as magenta hexagon in figure 12) of the SMC. The center of the density distribution shown here is very similar to the centroid (given in section 2.1) of our RC sample in 553 sub-regions which are used for the estimation of dereddened magnitude and depth.

As the data coverage of the OGLE III is not symmetric with respect to the density center, the radial density distribution of the RC stars within a radius of $0.8^{\circ}$ is obtained. The region within $0.8^{\circ}$ radius from the density center are divided into 16 equal area (28.3 square arcmin) annuli. The number of RC stars in each annuli is obtained and it is divided by the area of the annuli to obtain the number density. Thus the radial density distribution of the RC stars within $0.8^{\circ}$ radius from the density center is obtained and are shown as black squares in figure 13. The best fitted exponential disk profile and the King's profile are shown in the figure as green and blue solid lines respectively. We can see that the radial density profile of the RC stars in the SMC is marginally better described by the King's profile than by the exponential profile. We obtained the surface density profile of the $\mathrm{RC}$ stars in the whole observed area of the SMC. The number density of the RC stars in the 1280 sub-regions of the SMC are plotted against the radial distance of each sub-region from the density center in figure 13. The black points in the figure denotes the surface density of the RC stars in each sub-region. The best fit exponential disk profile and the King's profile are shown as green and blue dashed lines in figure 13. Here we can see that the surface density profile of the RC stars in the SMC is best described by the King's profile. The parameters obtained by fitting the radial density and surface density distributions by exponential and King's profiles are given in table 1. The estimated tidal radius of the SMC system is $\sim 7-12 \mathrm{kpc}$. Thus the RC stars in the SMC are

Table 1: Parameters of the Exponential and King's profiles

\begin{tabular}{lrrrrr}
\hline Data & $\mathrm{f}_{0 d}($ no/sq.kpc $)$ & $\mathrm{h}(\mathrm{kpc})$ & $\mathrm{f}_{0 k}(\mathrm{no} / \mathrm{sq} . \mathrm{kpc})$ & $\mathrm{r}_{c}(\mathrm{kpc})$ & $\mathrm{r}_{t}(\mathrm{kpc})$ \\
\hline Radial density distribution & $(104 \pm 0.4) \times 10^{3}$ & $1.87 \pm 0.1$ & $(141 \pm 7) \times 10^{3}$ & $1.08 \pm 0.02$ & $7 \pm 1$ \\
Surface density distribution & $(264 \pm 0.1) \times 10^{3}$ & $0.91 \pm 0.1$ & $(248 \pm 1) \times 10^{3}$ & $0.9 \pm 0.01$ & $12.04 \pm 0.01$ \\
\hline
\end{tabular}




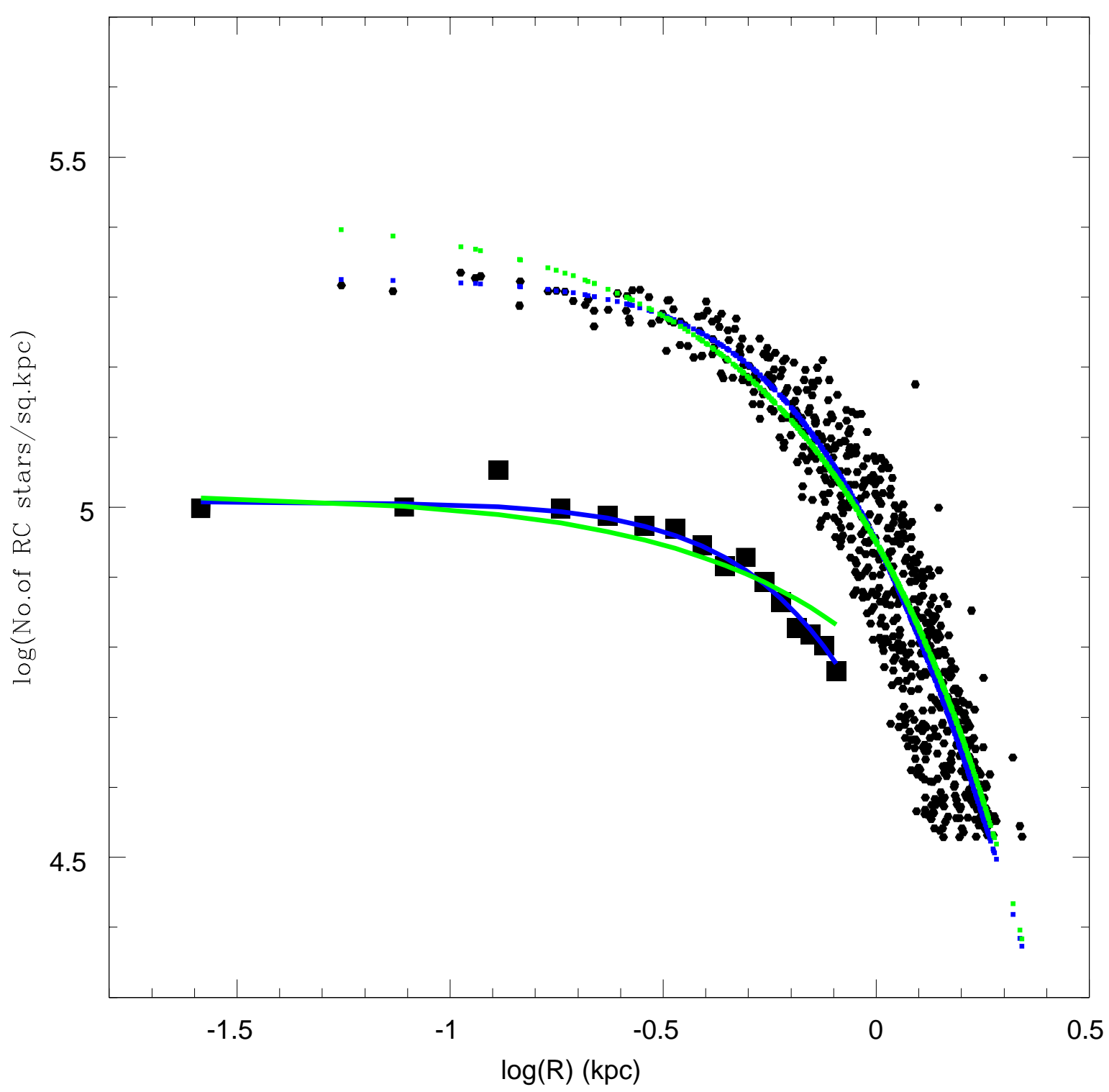

Fig. 13. - Radial density distribution of the RC stars within the radius of the $0.8^{\circ}$ in the SMC are shown as black squares. The best fitted King's profile and the exponential profile for the radial density distribution are shown as blue and green solid lines respectively. The surface density distribution of the RC stars for the whole observed region of the OGLE III are shown as black dots. The best fitted King's profile and the exponential profile for the surface density distribution are shown as blue and green dashed lines respectively. 
distributed in a spherical/ellipsoidal volume, which indicates that the SMC can be approximated as a spheroidal/ellipsoidal galaxy. But in the surface density profile we can see a spread in the points for each radii, which indicates that the volume in which the RC stars are distributed is not exactly spherical. Again from the map of the surface density distribution shown in figure 12, we can see the elongation in the NE-SW direction. Thus the RC stars in the SMC are distributed in an ellipsoidal system.

Our sample of RRLS which are pulsating in the fundamental mode consists 1904 stars. The density center of our sample is $\alpha=0^{h} 53^{m} 31^{s}, \delta=-72^{\circ} 59^{\prime} 15^{\prime \prime} .7$. The density center of our sample lies in between the two concentrations found by Soszyñski et al. (2010). To study the density profiles a large number of sample is required. The surface density map of the RRLS identified from the OGLE III photometric maps is shown in the lower panel of figure 7 in Soszyñski et al. (2010). They identified two concentrations in the RRLS spatial distribution and found that the RRLS in the SMC form a roughly circular strucutre in the sky which indicates that the RRLS in the SMC are distributed in a spheroidal/ellipsoidal volume.

\section{Axes ratio and orientation of the SMC ellipsoid}

We model the observed system of the SMC in which the RC stars and RRLS are distributed as a triaxial ellipsoid. The parameters of this ellipsoidal system, like the axes ratio and the orientation can be estimated using the inertia tensor analysis. The tensor analysis used here is similar to the

methods used by Pejcha \& Stanek (2009) and Paz et al. (2006), but with some modification. The tensor analysis used in the study is explained in appendix.

\subsection{RR Lyrae stars}

The method described in the appendix can be applied to our sample of the RRLS to estimate the parameters of the ellipsoidal component of the SMC. First we applied this method only to (x,y) system and found that the SMC RR Lyrae distribution is elongated with an axes ratio of 1:1.3 and the major axis has a position angle of $74^{\circ}(\mathrm{NE}-\mathrm{SW})$. We repeated the procedure to the $(\mathrm{x}, \mathrm{y}, \mathrm{z})$ coordinates of the RRLS. The axes ratio obtained is 1:1.3:6.47 and the longest axis is inclined with the line of sight direction with an angle $(i)$ of $0^{\circ} .4$. The position angle of the projection of the ellipsoid $(\phi)$ on the plane of the sky is given by $74^{\circ} .4$. The data of the RRLS used here contain the RRLS in the isolated north western OGLE III fields. As they are discrete points in the density distribution, we removed the RRLS in those fields and repeated the procedure. When we removed the RRLS in those fields, the density center changed to $\alpha=0^{h} 54^{m} 38^{s} .6, \delta=-73^{\circ} 4^{\prime} 52^{\prime \prime} .2$. The 
axes ratio obtained is 1:1.57:7.71 with $i=0^{\circ} .4$ and $\phi=66^{\circ} .0$. Here we can see that the coverage of the data plays an important role in the estimation of the structural parameters of the ellipsoid. This may also suggest that there may be a variation in the inner and outer structures of the SMC. Nidever et al (2011) found that the inner SMC $\left(\mathrm{R}<3^{\circ}\right)$ is more elliptical than the outer component $\left(3^{\circ}<\mathrm{R}<7^{\circ} .5\right)$.

In order to understand the radial variation of the inner structure we estimated the parameters using the data within different radii. At first, the analysis is done excluding the RRLS in the north western fields. The axes ratio, $i$ and $\phi$ of the RRLS distribution are obtained for the data within different radii, starting from $0.75^{\circ}$. The values obtained are given in table 2 . The values show that the $i$ is more or less constant with a value of around $0^{\circ} .5$. But the axes ratio and $\phi$ have a range. The axes ratio has a range from 1:1.05:19.84 to 1:1.57:7.7. The $\phi$ ranges from $60^{\circ}$ to $78^{\circ}$. The data within $0.75^{\circ}$ of the density center is symmetric and the parameters obtained confirm that the RRLS distribution in the SMC is slightly elongated in the NE-SW direction. As the radius increases the data coverage is not symmetric and circular, and the axis ratio of $\mathrm{x}$ and $\mathrm{y}$ show an increasing trend. The surface density map of RRLS shown (in figure 7 of Soszyñski et al. 2010) does not show large elongation, rather the map looks nearly circular with a mild elongation in the NE-SW direction. So the elongation obtained for the RRLS distribution in the plane of the sky, at larger radius where the data coverage is not even, may not be real. We did a similar analysis to understand the radial variation including the fields in the north western regions. Here the concentric circles with different radii are centered on the density center (given in last paragraph) estimated including the RRLS in the north western fields. These values are also given in table 2. From this also it is evident that there is a mild elongation in the NE-SW direction in the distribution of the RRLS in the SMC. Here we have to also keep in mind that the density center estimation of the SMC RRLS is not accurate as there are two concentrations found in the density distribution shown in figure 7 of Soszyñski et al. (2010). The variation in the density center also modifies the structural parameters of the distribution.

From the detailed analysis described in the last two paragraphs, it is clear that the quantitative estimates of the structural parameters of the SMC are very much dependent on the data coverage. Though there are differences in the values, we can say that the RR Lyrae distribution in the inner SMC is slightly elongated in the NE-SW direction. The longest axis Z', which is perpendicular to the X'Y' plane (a plane obtained by the counter clockwise rotation of the XY plane with an angle $\phi$ with respect to the $\mathrm{Z}$ axis) is aligned almost parallel to the line of sight, $\mathrm{Z}$ axis. The $\mathrm{x}, \mathrm{y}$ and $\mathrm{z}$ values of the whole sample of the RRLS are plotted in figure 14 to get a 3D visualization of the SMC structure. The figure clearly shows the elongation in the $Z^{\prime} \sim Z$ axis. 


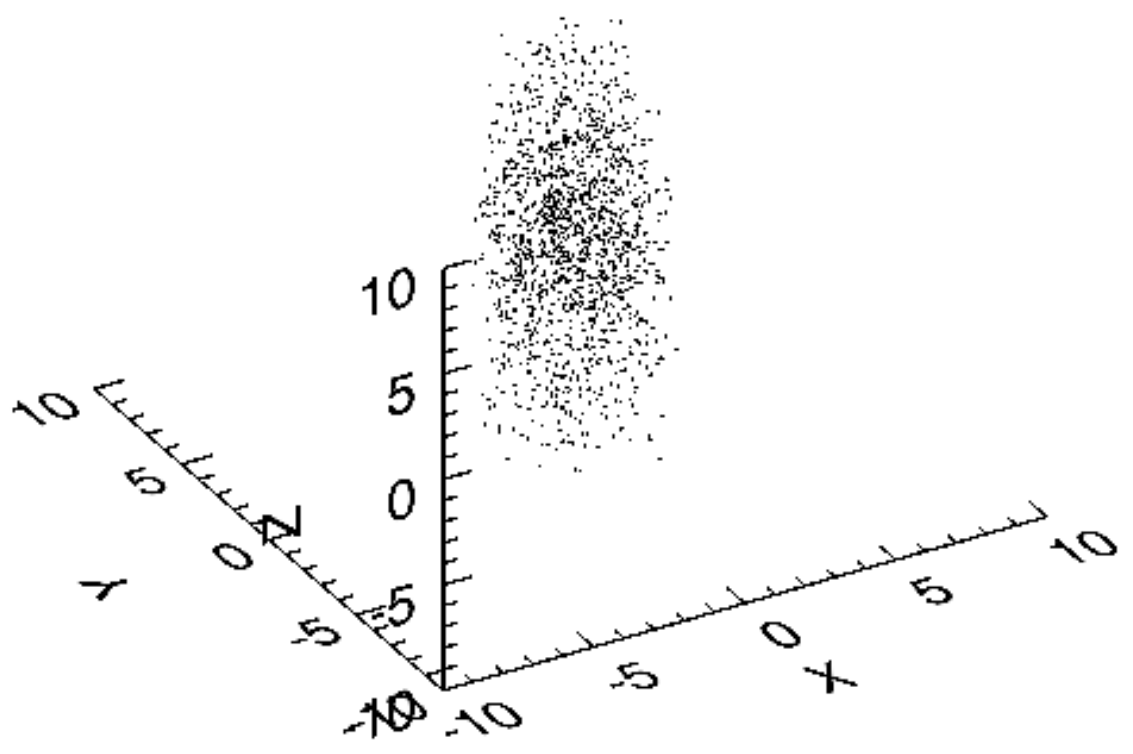

Fig. 14. - The $\mathrm{x}, \mathrm{y}$ and $\mathrm{z}$ values of the whole sample of the RRLS are plotted. 
Table 2: Orientation measurements of the ellipsoidal component of the SMC estimated using the RR Lyrae stars

Excluding the three north western fields

Center $\alpha=0^{h} 54^{m} 38^{s} .6, \delta=-73^{\circ} 4^{\prime} 52^{\prime \prime} .2$

\begin{tabular}{lrrrr}
\hline Data & No of RRLS & Axes ratio & $i$ & $\phi$ \\
\hline $\mathrm{r}<0^{\circ} .75$ & 421 & $1: 1.05: 19.84$ & $0^{\circ} .4$ & $78^{\circ} .83$ \\
$\mathrm{r}<1^{\circ} .00$ & 676 & $1: 1.03: 14.59$ & $0^{\circ} .4$ & $72^{\circ} .20$ \\
$\mathrm{r}<1^{\circ} .25$ & 924 & $1: 1.04: 11.35$ & $0^{\circ} .2$ & $75^{\circ} .68$ \\
$\mathrm{r}<1^{\circ} .50$ & 1187 & $1: 1.10: 9.43$ & $0^{\circ} .1$ & $63^{\circ} .41$ \\
$\mathrm{r}<1^{\circ} .75$ & 1407 & $1: 1.23: 8.66$ & $0^{\circ} .1$ & $61^{\circ} .68$ \\
$\mathrm{r}<2^{\circ} .00$ & 1563 & $1: 1.34: 8.21$ & $0^{\circ} .1$ & $66^{\circ} .00$ \\
$\mathrm{r}<2^{\circ} .25$ & 1711 & $1: 1.47: 7.87$ & $0^{\circ} .3$ & $67^{\circ} .62$ \\
$\mathrm{r}<2^{\circ} .50$ & 1780 & $1: 1.54: 7.73$ & $0^{\circ} .4$ & $67^{\circ} .48$ \\
$\mathrm{r}<2^{\circ} .75$ & 1798 & $1: 1.57: 7.71$ & $0^{\circ} .4$ & $66^{\circ} .30$ \\
$\mathrm{r}<3^{\circ} .00$ & 1803 & $1: 1.57: 7.71$ & $0^{\circ} .4$ & $65^{\circ} .96$ \\
\hline
\end{tabular}

Including the north western fields Center $\alpha=0^{h} 53^{m} 31^{s}, \delta=-72^{\circ} 59^{\prime} 15^{\prime \prime} .7$

\begin{tabular}{lrrrr}
\hline Data & No of RRLS & Axes ratio & $i$ & $\phi$ \\
\hline $\mathrm{r}<0^{\circ} .75$ & 428 & $1: 1.07: 20.01$ & $0^{\circ} .5$ & $48^{\circ} .84$ \\
$\mathrm{r}<1^{\circ} .00$ & 671 & $1: 1.03: 14.01$ & $0^{\circ} .4$ & $64^{\circ} .88$ \\
$\mathrm{r}<1^{\circ} .25$ & 927 & $1: 1.03: 11.19$ & $0^{\circ} .4$ & $67^{\circ} .84$ \\
$\mathrm{r}<1^{\circ} .50$ & 1177 & $1: 1.13: 9.59$ & $0^{\circ} .2$ & $59^{\circ} .54$ \\
$\mathrm{r}<1^{\circ} .75$ & 1399 & $1: 1.23: 8.70$ & $0^{\circ} .2$ & $60^{\circ} .10$ \\
$\mathrm{r}<2^{\circ} .00$ & 1568 & $1: 1.30: 8.00$ & $0^{\circ} .1$ & $64^{\circ} .87$ \\
$\mathrm{r}<2^{\circ} .25$ & 1755 & $1: 1.34: 7.22$ & $0^{\circ} .3$ & $68^{\circ} .78$ \\
$\mathrm{r}<2^{\circ} .50$ & 1845 & $1: 1.36: 6.89$ & $0^{\circ} .4$ & $70^{\circ} .80$ \\
$\mathrm{r}<2^{\circ} .75$ & 1892 & $1: 1.34: 6.57$ & $0^{\circ} .4$ & $73^{\circ} .70$ \\
$\mathrm{r}<3^{\circ} .00$ & 1904 & $1: 1.33: 6.47$ & $0^{\circ} .3$ & $74^{\circ} .40$
\end{tabular}




\subsection{Red Clump stars}

From the previous sections we found that the RC stars and RRLS occupy a similar volume of the SMC. Hence we can apply the method of inertia tensor to the RC stars for the estimation of the parameters of the ellipsoidal component of the SMC. First we apply this method to the (x,y) coordinates of the 553 sub-regions of the SMC. Here each $(x, y)$ pair represents the coordinates of a sub-region. We weighted it using the number of the RC stars identified in that region to get the axes ratio. We find that the RC distribution in the SMC is elongated with an axes ratio of $1: 1.48$ and the position angle of the major axis is $55^{\circ} .3$. Now we applied the same procedure to the $(\mathrm{x}, \mathrm{y}, \mathrm{z})$ system of the RC stars. In the case of RRLS, we took the distance of the individual RR Lyrae star with respect to the mean distance as the $\mathrm{z}$ coordinate. But in the case of RC stars we only have the mean magnitudes corresponding to the mean distances to the sub-regions. The mean magnitude of a region in the ellipsoidal or nearly spheroidal system is the average of the magnitude of symmetrically distributed RC stars. In order to get the real RC distribution we did the following. After subtracting the average extinction, we obtained the magnitude distribution of the RC stars for each sub-region. Using the method explained earlier in section 2.1, we estimated the peak and the width of the magnitude distribution for each sub-region and eventually the width corresponding to depth. The depth (front to back distance) of the RC stars in a region is the measure of the extend up to which the RC stars in that region is distributed. Most of the RC stars in a sub-region are at the mean distance obtained for that sub-region and the remaining are distributed within the depth of the region. We assume the distribution to be symmetric with respect to the mean distance to that sub-region. Initially we took the $\mathrm{I}_{0}$ values of the bins within 1-sigma width and converted them into $\mathrm{z}$ distances. As we have the number of stars in each bin we weighted and applied the inertia tensor analysis to the $\mathrm{x}, \mathrm{y}$ and $\mathrm{z}$ coordinates.

Earlier we found that the RC and RR Lyrae depth distributions are similar. Also, from figures 10 and 11 we can see that the RR Lyrae distribution extends up to 3-4 sigma depth. Hence we can take the $\mathrm{z}$ component to extend upto higher sigma levels (2-sigma, 3-sigma, 3.5-sigma, 4-sigma)

Table 3: Orientation measurements of the ellipsoidal component of the SMC using the RC stars

\begin{tabular}{lrrr}
\hline Data & Axes ratio & $i$ & $\phi$ \\
\hline RC stars within 1-sigma depth & $1: 1.49: 3.08$ & $4^{\circ} .22$ & $55^{\circ} .7$ \\
RC stars within 2-sigma depth & $1: 1.48: 5.26$ & $1^{\circ} .23$ & $55^{\circ} .4$ \\
RC stars within 3-sigma depth & $1: 1.48: 7.10$ & $0^{\circ} .68$ & $55^{\circ} .6$ \\
RC stars within 3.5-sigma depth & $1: 1.48: 7.88$ & $0^{\circ} .58$ & $55 .^{\circ} .5$ \\
RC stars within 4-sigma depth & $1: 1.48: 8.58$ & $0^{\circ} .49$ & $55 .^{\circ} .4$ \\
\hline
\end{tabular}


to understand the real RC distribution in the SMC. We applied the inertia tensor analysis using $\mathrm{z}$ components which extend up to 2-sigma, 3-sigma, 3.5-sigma \& 4-sigma. The parameters obtained are given in table 3. From the table we can see that the orientation measurements of the ellipsoidal component of the SMC using RC stars, where the $\mathrm{z}$ component extend up to 3.5 sigma is similar to the values obtained for the RR Lyrae distribution excluding the north western fields. As we removed the regions with RC stars less than 400, the north western fields are not included in the analysis. The axes ratio and the angle $i$ are matching well with the ellipsoidal parameters of the SMC, estimated using the distribution of the RRLS. The position angle, $\phi$ estimated using the RC stars and RRLS are slightly different.

Based on the analysis described above we can say that the RC stars in the SMC are distributed in an ellipsoidal system with an axes ratio of 1:1.48:7.88. The position angle, $\phi$ is $55^{\circ} .5$. The longest axis $Z^{\prime}$ which is perpendicular to the $\mathrm{X}^{\prime} \mathrm{Y}^{\prime}$ plane (obtained by the counter clockwise rotation of the $X Y$ plane with an angle $55^{\circ} .5$ with respect to the $\mathrm{Z}$ axis) is inclined with the $\mathrm{Z}$ axis with an angle of $0^{\circ} .58^{\circ}$. The inclination of the $Z^{\prime}$ axis with the line of sight is very small and we can assume the longest axis to be almost along the line of sight.

\subsection{Comparison of the structural parameters obtained from both the populations}

The quantities estimated in this analysis strongly depend on the data coverage. In order to compare the axes ratio and the orientation measurements obtained from both the populations, it is important to take the sample of both the population of stars from the same region. In the case of the RC stars regions which include the north western fields and also regions in the edges of the data set are omitted based on the selection criteria. Such a truncation of the data is not done in the case of the RRLS. Only the RRLS which are possible Galactic objects are removed from the analysis. These stars are not concentrated on a particular region but are scattered. If we remove the RRLS in the north western fields and the edges of the data, then the sample of RRLS cover the nearly the same region as the RC stars sample. We used the RRLS within the box of size size $-1.8-1.8$ in both the $\mathrm{X}$ and $\mathrm{Y}$ axes. Thus the RRLS in the edges of the data set and in the north western fields are

Table 4: Orientation measurements of the ellipsoidal component of the SMC using the RC stars and the RRLS in the same region. Excluding the north western fields and the edges of the data

\begin{tabular}{lrrr}
\hline Data & Axes ratio & $i$ & $\phi$ \\
\hline 1654 RRLS & $1: 1.46: 8.04$ & $0^{\circ} .50$ & $58^{\circ} .3$ \\
RC stars within 3.5-sigma depth & $1: 1.48: 7.88$ & $0^{\circ} .58$ & $55^{\circ} .5$ \\
\hline
\end{tabular}


nearly removed. Then we estimated the parameters using this sample of the RRLS and the values are given in table 4. From the table we can see that when the sample of both the populations are taken from similar regions of the SMC the estimated parameters match well.

\subsection{The actual structure of the SMC}

We find the $Z^{\prime} \sim Z_{\text {axis }}$ is the longest axis in the analysis of both the RRLS and the RC stars. The reason for such a result is mainly the coverage of the SMC. The studied data covers only the central regions thus restricting the coverage in the XY plane. On the other hand the full $\mathrm{Z}$ direction is sampled. Hence the present view of the SMC based on our axes ratio is like viewing only the central part of a sphere along the $\mathrm{Z}$ axis. Such a perspective will give an elongated $\mathrm{Z}$ axis. This is clear from figure 14. In order to test whether the $\mathrm{z}^{\prime} \sim \mathrm{z}$ axis is due to the viewing perspective, we have sampled the data similarly along the three axes. The extend of the sample along all the three axes can be made equal by taking the data only within a spherical radii $(\rho)$ of 2, 2.5 and 3 degrees from the center. We took the RRLS stars within these different radii and estimated the structural parameters. The estimated parameters are given in table 5. An elongation in the NE-SW axis is seen suggesting that the elongation of the RRLS in the NE-SW is a real feature. The decrease in the relative length of the $z^{\prime}$ axis is expected as the selection mentioned above removes relatively more number of stars along the $z^{\prime}$ direction than in the $x^{\prime}$ and y' directions. From table 5 we can see that the inclination of the longest axis with the line of sight axis decreases from inner to outer radii. The finite XY coverage restricts further analysis of this trend. The very small value of $i$ (given in tables 2, and 4) estimated from the analysis of all the RRLS, including those at larger $\mathrm{z}$ distances from the center, indicates that the decreasing trend of $i$ is continued to outer radii also. In table 5 we can also see an increase in $\phi$ and the relative length of y' axis from inner to outer region. From section 6.1 and from table 2 we can understand that these two quantities are dependent on the choice of the centroid of the system. So we cannot definitely say anything more about this trend. Another important result to be noticed from table 5 is that even though the $z$ ' axis is the longest of the three, the values of the longest and the second longest (y') axes are comparable. This suggests

Table 5: Estimated structural parameters with equal extent in all the three axes

\begin{tabular}{lrrrr}
\hline Data & Number of RRLS & Axes ratio & $\phi$ (degrees) & $i$ (degrees) \\
\hline$\rho<2.0$ & 344 & $1: 1.17: 1.28$ & 67.5 & 4.2 \\
$\rho<2.5$ & 540 & $1: 1.24: 1.39$ & 69.5 & 3.3 \\
$\rho<3.0$ & 730 & $1: 1.33: 1.61$ & 70.2 & 2.6 \\
\hline
\end{tabular}


that when the coverage along all the three axes becomes comparable, the structure of the SMC is spheroidal or slightly ellipsoidal.

The above analysis indicates that the XY extent of the SMC upto which stellar populations are studied/detected plays an important role in understanding the actual structure of the SMC and hence the estimation of the structural parameters. The tidal radius estimates give the radial extent up to which the stellar populations are bound to the SMC or up to which they are expected to be found. Gardiner \& Noguchi (1996) estimated the tidal radius of the SMC to be $\sim 5 \mathrm{kpc}$. Later Stanimirović et al. (2004) suggested the tidal radius of the SMC to be 4-9 kpc. The estimations obtained in our study from the radial density and surface density profiles of the RC stars, given in table 1, suggest the tidal radius to be 7-12 kpc. There are many recent studies which found old and intermediate stellar populations in the outer regions. Noël et al. (2007) found that up to 6.5 $\mathrm{kpc}$ from the SMC center, the galaxy is composed of both, intermediate-age and old population, without an extended halo. De Propris et al. (2010) estimated the SMC edge in the eastern direction to be around $6 \mathrm{kpc}$ from the survey of red giant stars in 10 fields in the SMC. A very recent

photometric survey of the stellar periphery of the SMC by Nidever et al (2011) found the presence of old and intermediate age populations at least up to $9 \mathrm{kpc}$. The above mentioned observational studies and tidal radius estimates suggest that the full extent of the SMC in the XY plane $(2 * 9$ $=18 \mathrm{kpc})$ is of the order of the front to back distance estimated $(14 \mathrm{kpc})$ along the $\mathrm{Z}$ axis. We found in earlier sections that the inner SMC is slightly elongated in the NE-SW direction. Thus we suggest, that the actual structure of the SMC is spheroidal or slightly ellipsoidal. Better estimates of the structural parameters can be obtained with a data set of larger sky coverage. In the following section, we combine previous studies and suggest an evolutionary model for the SMC.

\section{Discussion}

Bekki \& Chiba (2008) suggested that the SMC may be formed due to a dwarf-dwarf merger, resulting in a stellar spheroidal component and a gaseous disk component, happened before the formation of the MW-LMC-SMC system. They also suggest that since stellar populations formed before the merger event should have dynamically hot kinematics, the youngest age of stellar populations that show both spheroidal distributions and no or little rotation can correspond to the epoch when the merger occurred. As the merger event should evoke a large scale star formation event in the galaxy, the epoch of merger can also be traced from the enhancements seen in the star formation history of the SMC.

The most comprehensive study of the star formation history of the SMC is presented by Harris \& Zaritsky (2004). They derived the global star formation history of the SMC. They found that there was a significant epoch of star formation up to $8.4 \mathrm{Gyr}$ ago when $50 \%$ of the stars were 
formed, followed by a long quiescent period in the range $3 \mathrm{Gyr}<$ age $<8.4 \mathrm{Gyr}$, and a more or less continuous period of star formation starting $3 \mathrm{Gyr}$ ago and extending to the present. They also found three peaks in the SFR, at 2-3 Gyr, at $400 \mathrm{Myr}$, and $60 \mathrm{Myr}$ ago. Their CMDs do not go deep enough to derive the full star formation history from the information on the main sequence. Obtaining CMDs reaching the oldest main sequence turnoff is essential in order to properly constrain the intermediate-age and old population (Gallart et al. 2005). As we study the intermediate age and old stellar populations in the SMC, here we compare the star formation studies done based on the CMDs which reach the oldest main sequence turnoffs. These studies go deeper and hence have smaller field of views. Dolphin et al. (2001), Chiosi \& Vallenari (2007), and Noël et al. (2009) have done such studies and obtained the star formation history of the SMC.

Dolphin et al. (2001) found a broad peak of star formation between 5 and 8 Gyr ago. Chiosi \& Vallenari (2007) found two main episodes of star formation, at 300-400 Myr and between 3-6 Gyr. They also found that the star formation rate was low until $\approx 6$ Gyr ago, when only a few stars were formed. Noël et al. (2009) found star formation enhancements at two intermediate ages, a conspicuous one peaked at 4-5 Gyr old in all fields and a less significant one peaked at 1.5-2.5 Gyr old in all fields. The enhancement at old ages, with the peak at 10 Gyr old is seen in all fields. But in the western fields, this old enhancement is split into two at 8 Gyr old and at 12 Gyr old. Their farthest field is at $4.5 \mathrm{kpc}$. All these studies show an enhancement in the star formation in the SMC around 4-5 Gyrs ago.

If the 4-5 Gyr global star formation in the SMC is assumed to be due to a dwarf-dwarf merger, then according to the merger model, the merged galaxy will settle down within 1-3 Gyr (Lotz et al. 2008). This scenario will then demand a spheroidal component well mixed with stars older than 2 Gyr. Our results find that the old population (RR Lyrae stars, age $>9$ Gyr) and the intermediate age component (Red clump stars, age $=2-9$ Gyr) seem to occupy similar volume, suggesting that stars older than 2 Gyr are in a well mixed ellipsoid.

Tsujimoto \& Bekki (2009) proposed that the evidence of a major merger event in the SMC is imprinted in the age-metallicity relation as a dip in $[\mathrm{Fe} / \mathrm{H}]$. They predicted that the major merger with a mass ratio of 1:1 to 1:4 occurred at $~ 7.5 \mathrm{Gyr}$ ago in the SMC. Based on this model they could reproduce the abundance distribution function of the field stars in the SMC. As they could not correlate a peak in the star formation history of the SMC at the epoch of the merger they suggested that the major merger which occurred in the SMC at 7.5 Gyr ago did not trigger a major star burst due to some physical reasons but proceeded with a moderate star formation. Noël et al. (2009) did not find a dip in the age metallicity relation of the SMC, which Tsujimoto \& Bekki (2009) claim to detect and suggested as the imprint of a major merger at 7.5 Gyr ago. Again if this merger event in the SMC at 7.5 Gyr old is the reason for the kinematical and morphological differences of the young and old stars then we cannot expect stars younger than 4-5 Gyr in the 
spheroidal/ellipsoidal distribution. As we see the RC stars which have an age range of 2-9 Gyr in the spheroidal/ellipsoidal distribution we propose a merger event which started around 4-5 Gyr ago as the reason for the observed distribution of stars in the SMC.

A wider and larger future photometric (OGLE IV) and spectroscopic surveys which cover both the inner and outer regions of the SMC will help to understand the complete structure and kinematics of the SMC.

\section{Conclusions}

- The dereddened $\mathrm{I}_{0}$ magnitude of the RC stars and RRLS are used to determine the relative positions of the regions in the SMC with respect to the mean distance and it suggest that either the population of the RC stars and RRLS in the north eastern regions are different and/or the north eastern part of the SMC is closer to us.

- The line of sight depth of the SMC estimated using the RC stars and the RRLS is found to be $\sim 14 \mathrm{kpc}$. The depth profiles of both the population look similar indicating that these two populations are located in the similar volume of the SMC.

- The surface density distribution and the radial density profile of the RC stars suggest that they are distributed in a nearly spheroidal system. The tidal radius estimated for the SMC system is $\sim 7-12 \mathrm{kpc}$. An elongation from NE -SW is also seen in the surface density map of the RC stars in the SMC.

- The observed SMC is approximated as a triaxial ellipsoid and the structural parameters, like the axes ratio, inclination, $i$ of the longest axis with the line of sight and the position angle, $\phi$ of the projection of the ellipsoid on the plane of the sky are estimated using the inertia tensor analysis. From the analysis of the RC stars and RRLS in the same region of the SMC the parameters estimated turned out to be very similar. The estimated parameters are very much dependent on the data coverage.

- The study of data only within concentric spheres of radii 2, 2.5 and 3 degrees from the center, where the extent in all the three axes becomes equal, shows that the relative lengths of the $z$ ' and y' axes are comparable. We estimated an axes ratio of 1:1.33:1.61 with a $2^{\circ} .6$ inclination of the longest axis with the line of sight from the analysis of RRLS within 3 degrees in the X, Y and 
$\mathrm{Z}$ axes. The position angle of the projection of the ellipsoid on the sky obtained is $70^{\circ} .2$. Our tidal radius estimates and various observational studies of the outer regions suggest that the full extent of the SMC in the XY plane is similar to the front to the back distance estimated along the line of sight. These results suggest that the actual structure of the SMC is spheroidal or slightly ellipsoidal.

- We propose that the SMC experienced a merger with another dwarf galaxy at about 4-5 Gyr ago, and the merger process was completed in another 2-3 Gyr. This resulted in a spheroidal distribution comprising of stars older than 2 Gyr.

Smitha Subramanian acknowledges the financial support provided by Council of Scientific and Industrial Research (CSIR), India through SRF grant, 09/890(0002)/2007-EMR-I. The authors thank the OGLE team for making the data available in public. Thanks to Indu.G for her help in plotting figures. The authors thank the anonymous referee for the constructive suggestions which improved the manuscript.

\section{REFERENCES}

Bekki, K. \& Chiba, M. 2008, ApJ, 679, L89

Borissova, J., Rejkuba, M., Minniti, D., Catelan, M., \& Ivanov, V. D. 2009, A\&A, 502, 505

Caldwell, J. A. R. \& Coulson, I. M. 1985, MNRAS, 212, 879

-. 1986, MNRAS, 218, 223

Chiosi, E. \& Vallenari, A. 2007, A\&A, 466, 165

Cioni, M., Habing, H. J., \& Israel, F. P. 2000, A\&A, 358, L9

Cioni, M.-R.L., Girardi.L., Marigo.P., Habing, H.J., 2006, A\&A, 452, 195

Clementini, G., Gratton, R., Bragaglia, A., Carretta, E., Di Fabrizio, L., \& Maio, M. 2003, AJ, 125,1309

Cole, A. A. 1998, ApJ, 500, L137

Crowl, H. H., Sarajedini, A., Piatti, A. E., Geisler, D., Bica, E., Clariá, J. J., \& Santos, Jr., J. F. C. 2001, AJ, 122, 220 
De Propris, R., Rich, R. M., Mallery, R. C., \& Howard, C. D. 2010, ApJ, 714, L249

Dolphin, A. E., Walker, A. R., Hodge, P. W., Mateo, M., Olszewski, E. W., Schommer, R. A., \& Suntzeff, N. B. 2001, ApJ, 562, 303

Dopita, M. A., Lawrence, C. J., Ford, H. C., \& Webster, B. L. 1985, ApJ, 296, 390

Evans, C. J. \& Howarth, I. D. 2008, MNRAS, 386, 826

Gallart, C., Zoccali, M., \& Aparicio, A. 2005, ARA\&A, 43, 387

Gardiner, L. T. \& Hawkins, M. R. S. 1991, MNRAS, 251, 174

Gardiner, L. T. \& Noguchi, M. 1996, MNRAS, 278, 191

Girardi, L. \& Salaris, M. 2001, MNRAS, 323, 109

Graham, J. A. 1975, PASP, 87, 641

Grieve, G. R. \& Madore, B. F. 1986, ApJS, 62, 427

Groenewegen, M. A. T. 2000, A\&A, 363, 901

Harris, J. \& Zaritsky, D. 2004, AJ, 127, 1531

-. 2006, AJ, 131, 2514

Hatzidimitriou, D., Cannon, R. D., \& Hawkins, M. R. S. 1993, MNRAS, 261, 873

Hatzidimitriou, D., Croke, B. F., Morgan, D. H., \& Cannon, R. D. 1997, A\&AS, 122, 507

Haschke,R., Grebel.E.K., \& Duffau,S. 2011, AJ, 141

King, I. 1962, AJ, 67, 471

Lotz, J. M., Jonsson, P., Cox, T. J., \& Primack, J. R. 2008, MNRAS, 391, 1137

Maragoudaki, F., Kontizas, M., Morgan, D. H., Kontizas, E., Dapergolas, A., \& Livanou, E. 2001, A\&A, 379, 864

Massey, P., Lang, C. C., Degioia-Eastwood, K., \& Garmany, C. D. 1995, ApJ, 438, 188

Nidever, D.L., Majewski, S.R., Munoz, R.R., et al, 2011, ApJ, 733, L10

Noël, N. E. D., Aparicio, A., Gallart, C., Hidalgo, S. L., Costa, E., \& Méndez, R. A. 2009, ApJ, 705,1260 
Noël, N. E. D., Gallart, C., Costa, E., \& Méndez, R. A. 2007, AJ, 133, 2037

Olsen, K. A. G. \& Salyk, C. 2002, AJ, 124, 2045

Pagel B. E. J., Tautvaisiene G., 1998, MNRAS, 299, 535

Paz, D. J., Lambas, D. G., Padilla, N., \& Merchán, M. 2006, MNRAS, 366, 1503

Pejcha, O. \& Stanek, K. Z. 2009, ApJ, 704, 1730

Schlegel, D. J., Finkbeiner, D. P., \& Davis, M. 1998, ApJ, 500, 525

Sarajedini, A.1999, AJ, 118, 2321

Smith, H. A., Silbermann, N. A., Baird, S. R., \& Graham, J. A. 1992, AJ, 104, 1430

Soszyñski, I., Udalski, A., Szymañski, M. K., Kubiak, J., Pietrzyñski, G., Wyrzykowski, Ł., Ulaczyk, K., \& Poleski, R. 2010, Acta Astron, 60, 165

Soszyñski, I., Udalski, A., Szymanski, M., Kubiak, M., Pietrzynski, G., Wozniak, P., Zebrun, K., Szewczyk, O., \& Wyrzykowski, L. 2002, Acta Astron, 52, 369

Stanek, K. Z., Zaritsky, D., \& Harris, J. 1998, ApJ, 500, L141

Stanimirović, S., Staveley-Smith, L., \& Jones, P. A. 2004, ApJ, 604, 176

Subramaniam, A. 2003, ApJ, 598, L19

-. 2005, A\&A, 430, 421

-. 2006, A\&A, 449, 101

Subramanian, S. \& Subramaniam, A. 2009, A\&A, 496, 399

-. 2010, A\&A, 520, A24+

Suntzeff, N. B., Friel, E., Klemola, A., Kraft, R. P., \& Graham, J. A. 1986, AJ, 91, 275

Tosi, M., Gallagher, J., Sabbi, E., et al. 2008, IAUS, 255, 381

Tsujimoto, T. \& Bekki, K. 2009, ApJ, 700, L69

Udalski, A. 1998, Acta Astron, 48, 383 
Udalski, A., Soszyński, I., Szymański, M. K., Kubiak, M., Pietrzyński, G., Wyrzykowski, Ł., Szewczyk, O., Ulaczyk, K., \& Poleski, R. 2008, Acta Astron, 58, 329

van der Marel, R. P. \& Cioni, M. 2001, AJ, 122, 1807

van der Marel, R. P., Kallivayalil, N., \& Besla, G. 2009, The Magellanic System: Stars, Gas and Galaxies,, ed. J. T. van Loon \& J. M. Oliveira, IAU Symposium, 256, 81

Weinberg, M. D. \& Nikolaev, S. 2001, ApJ, 548, 712

Westerlund, B.E. 1997, The Magellanic Clouds, Cambridge (Cambridge Univ.Press)

Zaritsky, D., Harris, J., Grebel, E. K., \& Thompson, I. B. 2000, ApJ, 534, L53

\section{Appendix}

\section{Inertia tensor analysis}

Moment of inertia of a body characterizes the mass distribution within the body. For any rotating three dimensional system, we can compute the moment of inertia about the axis of rotation, which passes through the origin of a local reference (XYZ) frame, using the inertia tensor. The origin of the system is the center of mass of the body. Consider a system made up of i number of particles, each particle with a mass $\mathrm{m}$. For each particle the (x,y and z) coordinates with respect to the center of mass is known. Then, the moment of inertia tensor, I of the system is given by

where

$$
\mathrm{I}=\left(\begin{array}{ccc}
I_{x x} & I_{x y} & I_{x z} \\
I_{y x} & I_{y y} & I_{y z} \\
I_{z x} & I_{z y} & I_{z z}
\end{array}\right)
$$

$\mathrm{I}_{x x}=\Sigma_{i} \mathrm{~m}_{i}\left(\mathrm{y}_{i}^{2}+\mathrm{z}_{i}^{2}\right)$

$\mathrm{I}_{y y}=\Sigma_{i} \mathrm{~m}_{i}\left(\mathrm{x}_{i}^{2}+\mathrm{z}_{i}^{2}\right)$

$\mathrm{I}_{z z}=\Sigma_{i} \mathrm{~m}_{i}\left(\mathrm{x}_{i}^{2}+\mathrm{y}_{i}^{2}\right)$

$\mathrm{I}_{x y}=\mathrm{I}_{y x}=\Sigma_{i} \mathrm{~m}_{i}\left(\mathrm{x}_{i}+\mathrm{y}_{i}\right)$

$\mathrm{I}_{y z}=\mathrm{I}_{z y}=\Sigma_{i} \mathrm{~m}_{i}\left(\mathrm{y}_{i}+\mathrm{z}_{i}\right)$

$\mathrm{I}_{x z}=\mathrm{I}_{z x}=\Sigma_{i} \mathrm{~m}_{i}\left(\mathrm{x}_{i}+\mathrm{z}_{i}\right)$ 
The components, $\mathrm{I}_{x x}, \mathrm{I}_{y y}$ and $\mathrm{I}_{z z}$ are called the moments of inertia while $\mathrm{I}_{x y}, \mathrm{I}_{y x}, \mathrm{I}_{x z}, \mathrm{I}_{z x}, \mathrm{I}_{y z}$ and $\mathrm{I}_{z y}$ are the products of inertia. These components given above are basically specific to the local reference frame and reflect the mass distribution within the system in relation to the local reference frame. If we align the axes of the local reference frame in such a way that the mass of the system is evenly distributed around the axes then the product of inertia terms vanish. This would mean the transformation of the local reference frame (XYZ) to a system (X'Y'Z'). In the new frame of reference, inertia tensor, I' is given by

$$
I^{\prime}=\left(\begin{array}{ccc}
I_{x^{\prime} x^{\prime}} & 0 & 0 \\
0 & I_{y^{\prime} y^{\prime}} & 0 \\
0 & 0 & I_{z^{\prime} z^{\prime}}
\end{array}\right)
$$

The terms $\mathrm{I}_{x^{\prime} x^{\prime}}, \mathrm{I}_{y^{\prime} y^{\prime}}$ and $\mathrm{I}_{z^{\prime} z^{\prime}}$ are the non zero diagonal terms of the inertia tensor in the new reference frame ( $X^{\prime} Y^{\prime} Z^{\prime}$ ) and are called the principal moments of inertia of the body. The three axes in the new reference frame are called the principal axes of the body.

To determine the principal axes of the system we have to diagonalize the inertia tensor I, which is obtained with respect to the local reference frame. The diagonalization of the inertia tensor provides three eigen values $\left(\lambda_{1}, \lambda_{2}\right.$ and $\left.\lambda_{3}\right)$ which correspond to the moments of inertia ( $\mathrm{I}_{x^{\prime} x^{\prime}}, \mathrm{I}_{y^{\prime} y^{\prime}}$ and $\mathrm{I}_{z^{\prime} z^{\prime}}$ ) about the principal axes. For each eigen value value we can compute the corresponding eigen vector. The eigen vectors corresponding to each eigenvalues are given as $\mathrm{e}_{\lambda_{1}}$ $=e_{11} i^{\prime}+e_{12} j^{\prime}+e_{13} k^{\prime}, e_{\lambda_{2}}=e_{21} i^{\prime}+e_{22} j^{\prime}+e_{23} k^{\prime} \& e_{\lambda_{3}}=e_{31} i^{\prime}+e_{32} j^{\prime}+e_{33} k^{\prime}$ where i', j' and $k^{\prime}$ are unit vectors along the $X^{\prime}, Y^{\prime}$ and $Z^{\prime}$ axes respectively. The transformation of the $X Y Z$ system to $X^{\prime} Y^{\prime} Z^{\prime}$ can be obtained using the transformation matrix, T which is made up of the 9 components of the 3 eigen vectors. The transformation matrix, $\mathrm{T}$ is given by

$$
\mathrm{T}=\left(\begin{array}{lll}
e_{11} & e_{21} & e_{31} \\
e_{12} & e_{22} & e_{32} \\
e_{13} & e_{23} & e_{33}
\end{array}\right)
$$

From the eigen values and the transformation matrix, the axes ratio and the orientation of the characteristic ellipsoid that best describes the spatial distribution of the particles in the system can be obtained. As the moment of inertia of a system characterizes the resistance of the system to rotation, the component of moment of inertia along the major axis of the system will be least and maximum along the minor axis. Thus the three eigen values which represent the moments of inertia of the three axes (such that $\mathrm{I}_{x^{\prime} x^{\prime}}>\mathrm{I}_{y^{\prime} y^{\prime}}>\mathrm{I}_{z^{\prime} z^{\prime}}$ ) of the ellipsoid can be written as

$\mathrm{I}_{x^{\prime} x^{\prime}}=\mathrm{M}\left(\mathrm{a}^{2}+\mathrm{b}^{2}\right)$

$\mathrm{I}_{y^{\prime} y^{\prime}}=\mathrm{M}\left(\mathrm{a}^{2}+\mathrm{c}^{2}\right)$

$\mathrm{I}_{z^{\prime} z^{\prime}}=\mathrm{M}\left(\mathrm{b}^{2}+\mathrm{c}^{2}\right)$ 
where $a, b$ \& $c$ are the semi-axes of the ellipsoid such that $a>b>c$ and $M$ the total mass of the system. Using the above relations we can estimate the axes ratio of the ellipsoid which best describes the spatial distribution of the particles in the system. The transformation matrix, $T$ describes the spatial directions or the orientation of the ellipsoid with respect to the local reference frame. 